\title{
Recent Developments in the Design, Capabilities and Autonomous Operations of a Lightweight Surface Manipulation System and Test-bed
}

\author{
John T. Dorsey ${ }^{1}$, Thomas C. Jones ${ }^{2}$, William R. Doggett ${ }^{3}$, Jeffrey S. Brady ${ }^{4}$, Felecia C. Berry ${ }^{5}$, and George G. \\ Ganoe $^{6}$ \\ NASA Langley Research Center, Hampton, VA, 23681
}

\author{
Eric J. Anderson ${ }^{7}$ \\ Purdue Universtiy, West Lafayette, IN, 47907 \\ Bruce D. King ${ }^{8}$ \\ Lockheed Martin, Hampton, VA, 23681 \\ and \\ C. David Mercer ${ }^{9}$ \\ Stinger Ghaffarian Technologies, Hampton, VA, 23666
}

\begin{abstract}
The first generation of a versatile high performance device for performing payload handling and assembly operations on planetary surfaces, the Lightweight Surface Manipulation System (LSMS), has been designed and built. Over the course of its development, conventional crane type payload handling configurations and operations have been successfully demonstrated and the range of motion, types of operations and the versatility greatly expanded. This enhanced set of $\mathbf{1}^{\text {st }}$ generation LSMS hardware is now serving as a laboratory test-bed allowing the continuing development of end effectors, operational techniques and remotely controlled and automated operations. This paper describes the most recent LSMS and test-bed development activities, that have focused on two major efforts. The first effort was to complete a preliminary design of the $2^{\text {nd }}$ generation LSMS that has the capability for limited mobility and can reposition itself between lander decks, mobility chassis, and fixed base locations. A major portion of this effort involved conducting a study to establish the feasibility of, and define, the specifications for a lightweight cable-drive waist joint. The second effort was to continue expanding the versatility and autonomy of large planetary surface manipulators using the $1^{\text {st }}$ generation LSMS as a test-bed. This has been accomplished by increasing manipulator capabilities and efficiencies through both design changes and tool and end effector development. A software development effort has expanded the operational capabilities of the LSMS test-bed to include; autonomous operations based on stored paths, use of a vision system for target acquisition and tracking, and remote command and control over a communications bridge.
\end{abstract}

\footnotetext{
${ }^{1}$ Senior Research Engineer, Structural Mechanics and Concepts Branch, MS-190, Associate Fellow, AIAA.

${ }^{2}$ Research Engineer, Structural Mechanics and Concepts Branch, MS-190, Member, AIAA.

${ }^{3}$ Senior Research Engineer, Structural Mechanics and Concepts Branch, MS-190, Member, AIAA.

${ }^{4}$ Aerospace Engineer, Mechanical Systems Branch, MS-432.

${ }^{5}$ Electronics Engineer, Advanced Sensing \& Optical Measurement Branch, Mail Stop 493.

${ }^{6}$ Computer Engineer, Electromagnetics \& Sensors Branch, MS-473.

${ }^{7}$ Graduate Student, Mechanical Engineering Department, 585 Purdue Mall.

${ }^{8}$ Senior Designer, Aeronautics Systems Engineering Branch, MS-238.

${ }^{9}$ Application Developer, Structural Mechanics and Concepts Branch, MS-190.
} 


\section{Nomenclature}

API = Application Programming Interface

GSFC $=$ Goddard Space Flight Center

GUI = Graphical User Interface

HRS = Human Robotic Systems

$J P L=$ Jet Propulsion Laboratory

LaRC = Langley Research Center

LSMS = Lightweight Surface Manipulation System

$M C C=$ Multi-Center Cockpit

$P^{3} E \quad=$ Passive Pose Position Engine

PIGI = Predictive Interactive Graphical Interface

RAPID = Robot Application Programming Interface Delegate

SBIR = Small Business Innovative Research

$T C P=$ Transmission Control Protocol

$U D P=$ User Datagram Protocol

$V L L L \quad=$ Variable-Length Lifting Link

\section{Introduction}

$\mathrm{T}$ he first generation of a versatile high performance device for performing payload handling and assembly operations on planetary surfaces, the Lightweight Surface Manipulation System (LSMS), has been designed and built. Conventional crane type payload handling configurations and operations were successfully demonstrated by the LSMS during field tests at Moses Lake, Washington ${ }^{1,2}$, as shown in Figure 1. It is critical that the device be versatile and able to accomplish a variety of tasks due to the large investment required to develop and test a device for planetary surface operations. Thus, following initial testing, the range of motion, types of operations and the versatility of the LSMS were greatly expanded to include; operations in forklift mode, side grappling of payloads, digging and positioning of loose regolith, and performing a variety of tasks using a set of specialized tools and end effectors $^{3}$. This updated set of $1^{\text {st }}$ generation LSMS hardware is now serving as a laboratory test-bed and is being used to continue to develop end effectors, operational techniques and remotely controlled and automated operations.

The viability of the LSMS is dramatically increased if it includes a limited capability for self-mobility that allows it to offload itself from the lander and onto a planetary surface (or a mobility chassis) and subsequently reposition itself at other outpost locations. Initial work to develop a $2^{\text {nd }}$ generation LSMS, featuring this capability to self-offload, resulted in preliminary designs for two of the key mechanical components; lightweight truss links and a mechanically-driven waist joint $^{3}$. The 2nd generation LSMS is

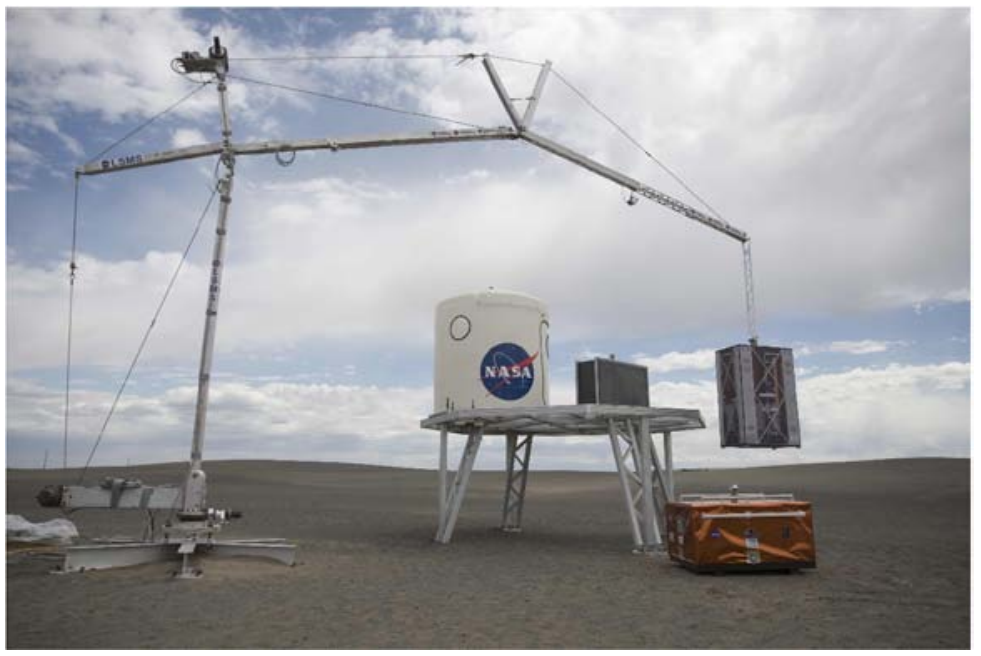

Figure 1. Payload handling operations performed at Moses Lake field test. specified to have longer reach and three times the payload capability of the current test-bed, yet by incorporating design improvements and refinements, its mass is predicted to be less than that of the current device ${ }^{3}$.

The most recent LSMS and test-bed development activities have focused on two major efforts. The first is to complete a preliminary design of the $2^{\text {nd }}$ generation LSMS. A major potion of this effort involved conducting a study to establish the feasibility of and define the specifications for a lightweight cable-drive waist joint. The second effort was to continue expanding the versatility and autonomy of large planetary surface manipulators using the $1^{\text {st }}$ generation LSMS as a test-bed. This paper will describe and summarize the results of the developments associated with these two efforts. 


\section{Design of a 2nd generation LSMS}

The LSMS will initially arrive at a planetary surface attached to the top of a lander deck, and from there, unload payloads and equipment to the planetary surface. It would not be either mass or cost effective if each lander had to include a LSMS for unloading operations. In addition, the capability to move and manipulate payloads and regolith, and perform inspection and repair operations will be required at many locations on the planetary surface for the lifetime of the outpost. Thus, the operational viability of the LSMS strongly depends on it being a repositionable and mobile system with the ability to relocate its base from its initial position on the lander onto a mobility chassis or fixed locations around the planetary outpost. This would allow the LSMS to unload subsequent cargo landers as they arrived, for example. The primary goal of the 2nd generation design effort is to incorporate this self-offload capability and complete the hardware design of all necessary supporting components. The team has also established a performance goal; that the 2nd generation LSMS mass be no greater than that of the 1st LSMS.

In Reference 3 , the performance specifications of the $1^{\text {st }}$ and $2^{\text {nd }}$ generation implementations of the LSMS are compared. Pertinent information that compares the two implementations and highlights the increased performance and reduced mass is summarized in Table 1. The reference also describes the changes made to the tension cable arrangement required to allow the LSMS to support itself from the wrist (with the base released and elevated), and modifications to the spreaders required to allow full range of motion. Finally, the reference summarizes a preliminary design for the truss links and an initial concept for a lightweight cable-drive waist joint.

Table 1. Comparison of $1^{\text {st }}$ and $2^{\text {nd }}$ generation LSMS capabilities.

\begin{tabular}{|l|c|c|c|c|c|c|c|}
\hline $\begin{array}{c}\text { Design } \\
\text { Identifier }\end{array}$ & Height, $\mathrm{m}$ & $\begin{array}{c}\text { Reach at } \\
\text { Wrist, } \mathrm{m}\end{array}$ & $\begin{array}{c}\text { Reach at } \\
\text { Elbow, } \mathrm{m}\end{array}$ & $\begin{array}{c}\text { Lunar } \\
\text { Payload - } \\
\text { Wrist, kg }\end{array}$ & $\begin{array}{c}\text { Lunar } \\
\text { Payload - } \\
\text { Elbow, kg }\end{array}$ & $\begin{array}{c}\text { Device } \\
\text { Mass, kg }\end{array}$ & $\begin{array}{c}\text { Construction } \\
\text { Type }\end{array}$ \\
\hline $1^{\text {st }}$ Gen. & 3.75 & 7.5 & 3.76 & 1,000 & 1,742 & 309 & Al. tube \\
\hline $2^{\text {nd }}$ Gen. & 4.25 & 8.5 & 4.26 & 3,000 & 5,226 & 190 & Al. truss \\
\hline
\end{tabular}

This section describes the subsequent work that was completed, resulting in a preliminary design package for the complete $2^{\text {nd }}$ generation LSMS. The complete set of features, changes and improvements made in evolving the LSMS from its $1^{\text {st }}$ to its $2^{\text {nd }}$ generation implementation is summarized. A single-link implementation of the $2^{\text {nd }}$ generation design that was configured for planetary surface crew operations is also described.

\section{A. Summary of New Features}

An oblique view of the $2^{\text {nd }}$ Generation LSMS is shown in the center of Figure 2a. Around the perimeter of Figure 2a are images highlighting new design features of the $2^{\text {nd }}$ generation device. The overall mechanical design architecture of the $2^{\text {nd }}$ generation LSMS is nearly identical to the first generation design architecture with the exception of how the actuator cable that controls the elbow is routed to enable the wrist to support the LSMS mass during self off load.

To prevent cable contact between the elbow control cable and the arm support cable, the arm support cable was divided into 2 separate lines as depicted in upper right of Figure 2a. The dual support cables provide additional torsion stability to the arm and a weight advantage because the cable can be attached directly to the exterior cross section of the arm instead of at a fitting in the center of the arm cross section. The elbow control cable now passes over a pulley as shown in the upper left of the figure near the shoulder to enable the motor for elbow control to be located at the base of the device.

The desire to have all motors located at the base of the device, lower right of Figure 2a, enables the $2^{\text {nd }}$ generation LSMS to be controlled manually from the base in the event of an electronics failure, including power failure, control cabling failure, sensor failure or motor failure. The device is designed to be operated open loop using either a hand held drill or manual ratchet fitted with a drive socket which engages the hoist shaft directly. Thus an EVA astronaut could actuate the LSMS manually in a contingency situation. An additional benefit of having the motors at the base is that it significantly simplifies access for repair of replacement of these components. Also visible is an additional motor to enable the king post to lean forward, toward the wrist, to increase reach and support shovel operations.

To support automated self off-load and leveling, three electrically driven leveling studs are used in the $2^{\text {nd }}$ generation unit, as shown in the lower left of Figure 2a. As with the primary drive motors, these units can be manually operated if necessary. During a self off-load maneuver, the wrist grapples a hard point so that the base can 
be disengaged. This connection to the hard point is enabled by a tool having 3 driven studs arranged in an identical manner to that shown at the base, thus enabling base and wrist hard point locations to be used interchangeably.

Figure $2 \mathrm{~b}$ depicts the LSMS deployment sequence from its compact packaged state. As in the $1^{\text {st }}$ generation LSMS, the $2^{\text {nd }}$ generation LSMS deploys using the same motors required for articulation. Deployment starts with the $2^{\text {nd }}$ generation LSMS vertical as shown on the lower left and progresses clockwise in the figure to the deployed configuration shown on the lower right. First, the s1 spreader deploys and locks in place followed by activation of the shoulder motor to raise the arm and lock it into place at the top of the kingpost. In the $2^{\text {nd }}$ generation

implementation, the king post and arm are not rigidly attached initially as was done in the $1^{\text {st }}$ generation design. Connection between the king post and arm occurs during deployment, significantly reducing the offset between the centerline of the arm cross section and the shoulder axis in operation, improving the

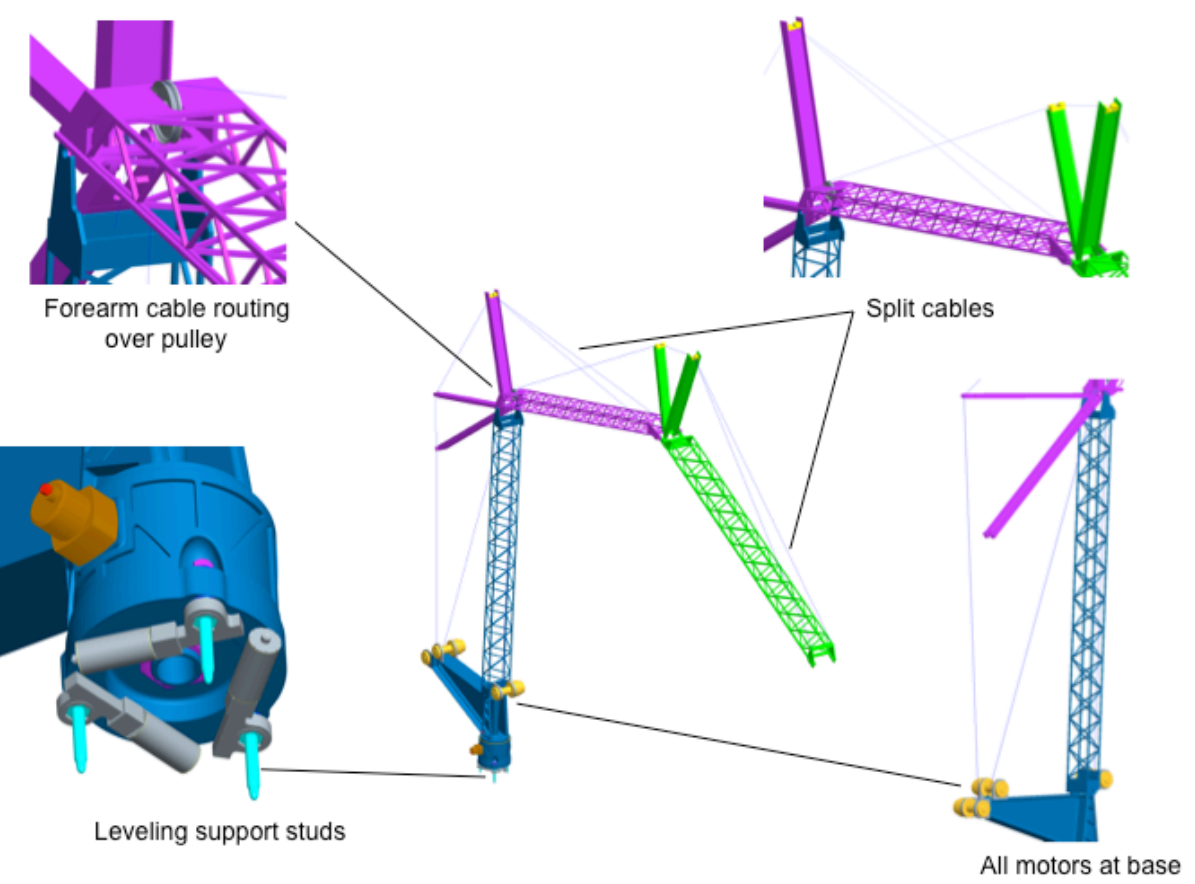

a. Oblique view of $2^{\text {nd }}$ generation LSMS highlighting new features.

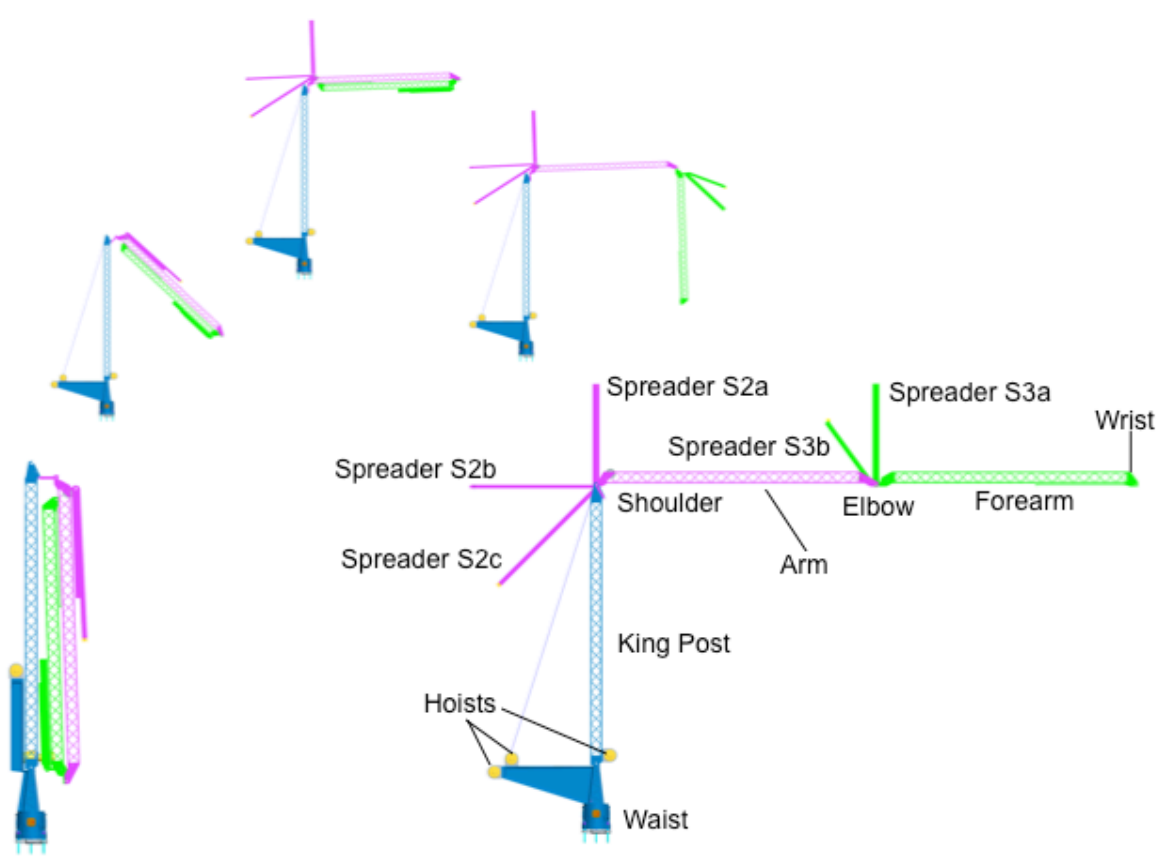

b. Deployment sequence of $2^{\text {nd }}$ generation LSMS.

efficiency of the LSMS and reducing its weight. Next the shoulder spreaders (s2c, s2b, s2a) are deployed followed by the elbow spreaders (s3b, s3a) and forearm.

\section{B. Lightweight Cable-Drive Waist Joint Feasibility}

In order to establish the feasibility of the lightweight cable-drive waist joint concept described in Reference 3, two contractors (RIETech and Honeybee Robotics Inc.) were awarded independent study contracts. In order to define performance specifications for the study, a single link version of the LSMS, as shown in Figure 3a, was 
specified. Figure 3b depicts a conceptual model of such a drive $\mathrm{e}^{3}$, where the capstan is driven by a motor through a gear train and where the gear train allows the unit to back-drive if a predetermined torque limit is reached. Cables wound around the capstan transfer torque from the motor to the driven ring and the ring causes the LSMS to rotate at its waist.

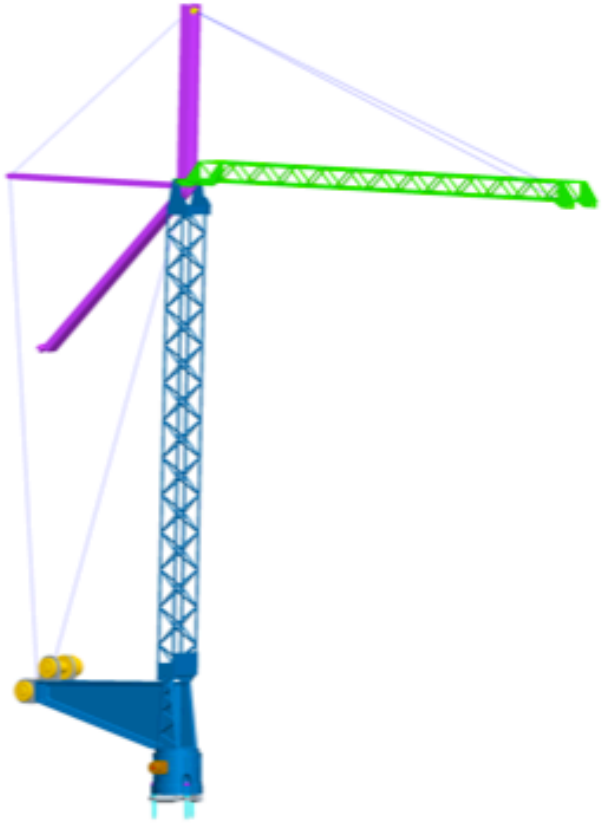

a. Single-link $2^{\text {nd }}$ generation design.

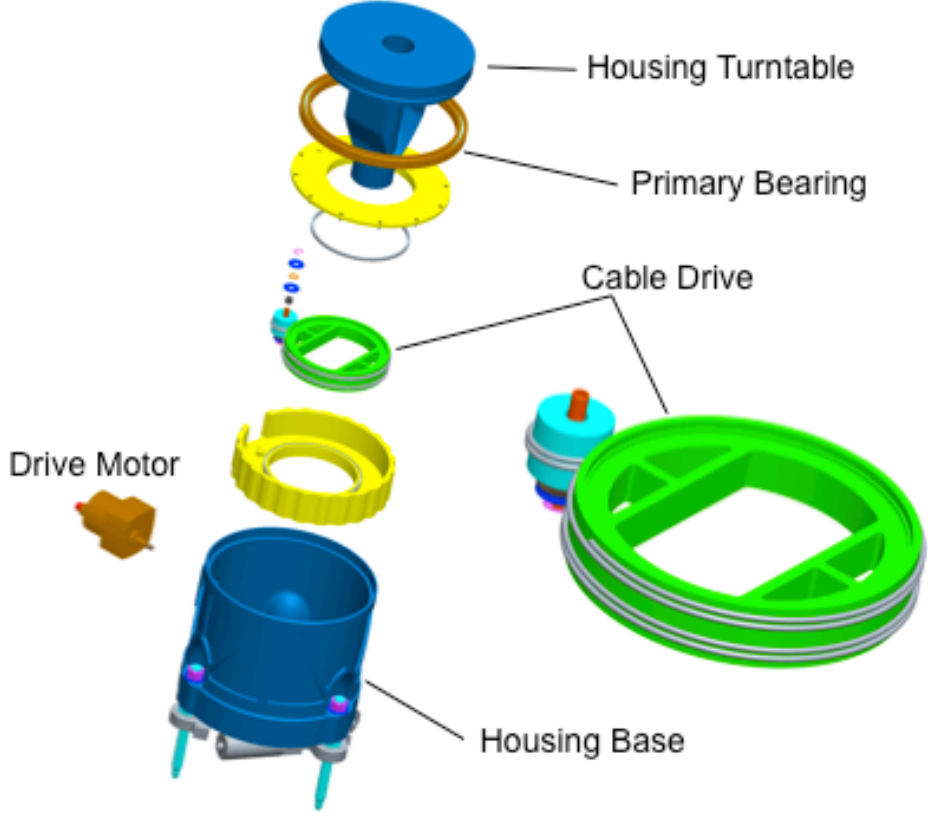

b. Cable drive waist joint concept.

Figure 3. Single link LSMS and cable-drive waist joint concept.

Each study contractor was asked to develop a recommended design and provide a detailed description of that design as well as the analysis approach used and results of the analyses. Important design factors that were addressed included; mechanical dimensions, drive ratio of capstan/driven ring and of gear box, torque output \& torque slip approach, deflection under load (backlash), assembly approach, mechanical loads, system weight, failure modes, and cable lifetime. In order to select a design, they were to consider the following metrics; unit mass, unit diameter, amount of backlash, capability for manual drive (as backup if motor fails), torque slip approach and ability to adjust critical torque, system lifetime, system maintenance schedule and rotation positioning precision. Specific requirements for the desired waist joint were provided for the study and are summarized in Table 2.

Honeybee Robotics developed analytical formulas that were used to size the various components of the capstan drive mechanism, generating data to support a preliminary design. The mechanism layout that they proposed for the drive system is shown in Figure 4. The study determined specifications for the following quantities: cable preload, number of cable wraps around the input and output drums, drive stiffness, torque limits, gear box type, amount of drive backlash, and motor selection. The height of the drive unit depends on the number of cable wraps on the output drum, which is a function of the cable diameter, which in turn, is a function of the maximum cable tension. Three feasible designs were developed; for 2.0, 3.0 and 5.0 factors of safety on the cable ultimate strength, giving cable stack heights of $72 \mathrm{~mm}, 83 \mathrm{~mm}$ and $124 \mathrm{~mm}$ respectively. The estimated mass for the complete joint system was $342 \mathrm{lb}$, which includes a 20 percent margin.

RIEtech performed analyses to determine if their proprietary Roto-Lok ${ }^{\circledR}$ drive design could be customized to meet the requirements of the LSMS waist joint. REItech developed a preliminary set of drive specifications based on the requirements in Table 2, augmented with additional inputs (based on their engineering experience) specific to their drive design. The specifications served as the input data to REItech's classical analysis model (Roto-Lok® Analysis Software Version 5.0). The analysis showed that a Roto-Lok ${ }^{\circledR}$ drive could be successfully sized to fit within the conceptual LSMS waist joint volume (see Figure 3b). Figure 5 illustrates the mechanical dimensions and the configuration of the proposed drive. With the Roto-Lok ${ }^{\circledR}$ drive, a preloaded cable is wrapped around the capstan and the main drum. A motor turns the capstan, which, through the wound cable, turns the drum (which is a component of the waist joint). Figure 5a shows details of the groove configuration and drive dimensions, while 
Figure $5 b$ depicts the entire drive configuration. Suggestions were also made for further refinements to the design that would increase both the drive stiffness and its lifetime. In summary, two independent studies have established the viability of a lightweight cable drive waist joint that meets LSMS performance requirements.

Table 2. Cable-drive waist joint design requirements.

\begin{tabular}{|c|c|c|}
\hline Requirement & $\begin{array}{r}\text { Value } \\
\end{array}$ & Notes/Remarks \\
\hline Mass & $<179 \mathrm{~kg}$ (395 lbs) & \\
\hline Diameter & $<45.7 \mathrm{~cm}$ (18 inches) & \\
\hline Volume & & Tracked, not specified. \\
\hline Torque: Operational & $2,260 \mathrm{~N}-\mathrm{m}(20,000$ in-lbf) & $\begin{array}{l}\text { 4,520 N-m (40,000 in-lbf) ultimate } \\
\text { (failure) torque }\end{array}$ \\
\hline Torque: Slip & 2,825 N-m (25,000 in-lbf) & \\
\hline Rotation Rate: No Payload & 0.3 degrees/second & \\
\hline Rotation Rate: Max Payload & 0.1 degrees/second & \\
\hline Rotation Range & \pm 180 degrees & \pm 360 degrees desired \\
\hline Brake Torque & $>2,825 \mathrm{~N}-\mathrm{m}$ (25,000 in-lbf) & $\begin{array}{l}\text { One primary and one redundant } \\
\text { brake. }\end{array}$ \\
\hline Position Precision & 20 arc-seconds & \\
\hline Safety Factor: Cable Strength & $2.0-3.0$ (minimum) & $4.0-5.0$ (desired) \\
\hline Duty Cycle & $\begin{array}{l}\text { 10s of revolutions of output shaft per } \\
\text { week }\end{array}$ & \\
\hline Power & From 15 amp 120 Volt AC & \\
\hline Operating temperature & $\begin{array}{l}-6.7 \text { to } 48.9 \text { degrees } \mathrm{C}(20-120 \\
\text { degrees } \mathrm{F})\end{array}$ & \\
\hline $\begin{array}{l}\text { Factor of Safety for mechanical } \\
\text { components }\end{array}$ & 2.0 for yield, 3.0 for ultimate & \\
\hline Manual Drive Mode & & Using wrench or handle \\
\hline
\end{tabular}

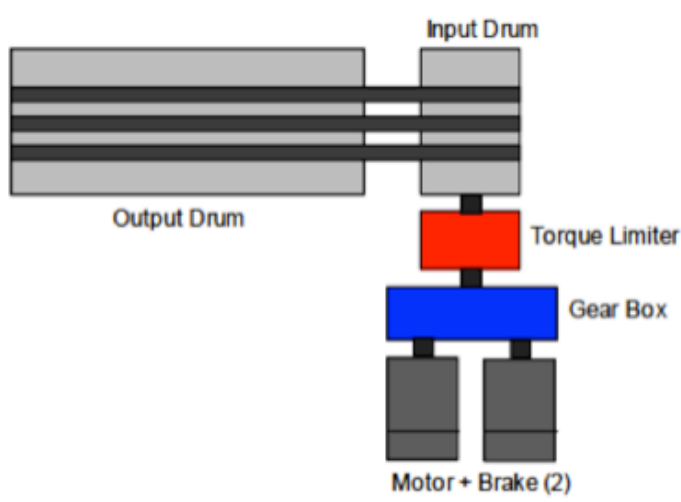

a. Waist drive mechanism layout.

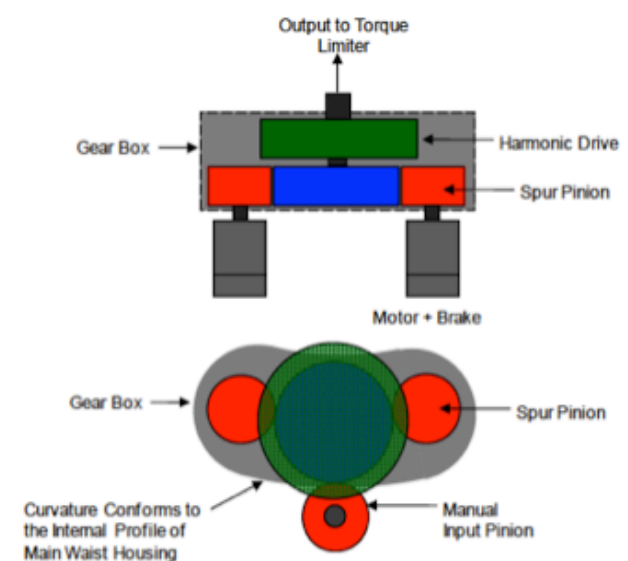

b. Gearbox layout.

Figure 4. Honeybee waist joint drive system. 


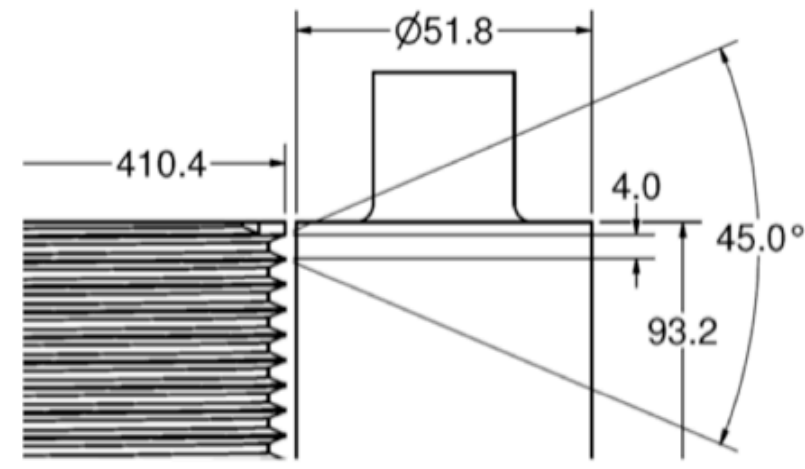

a. Capstan and drum dimensions.

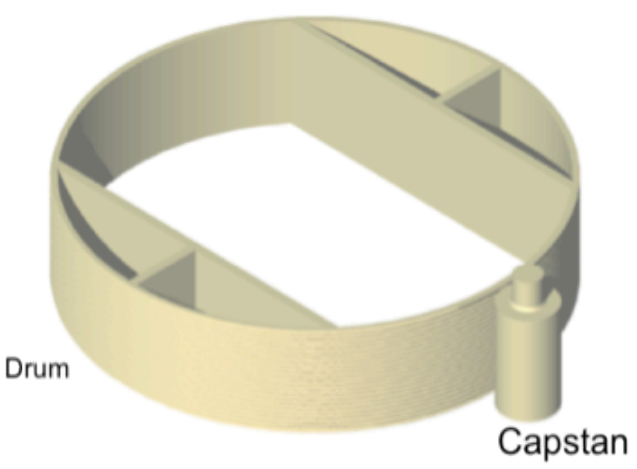

b. Capstan and drum rendering.

Figure 5. RIETech waist joint configuration.

\section{Single-Link Crew Operations Version of LSMS}

A conceptual design was also developed for a single-link version of the $2^{\text {nd }}$ generation LSMS. The focus mission for this version was to support the critical need for lifting incapacitated crew from a planetary surface, to the top of a lander deck, allowing the injured crew to be positioned inside an ascent stage for either emergency medical care or evacuation. Figure 6 depicts one implementation of the hardware for such a scenario. Having a single link LSMS on board the lander for crewed flights would offer the additional advantages of serving as a crew elevator and providing a means to easily lift and lower equipment and scientific samples from the planetary surface to the ascent stage and lander deck. If the mass of the single link LSMS for this application is sufficiently small, transporting a single link LSMS on every crewed lander would not be considered a penalty.

In order to size the single link LSMS, two payload scenarios were considered. In the first, a crew rescue basket is retrieved from its storage location on the lander, lowered to the surface where two crew members (the

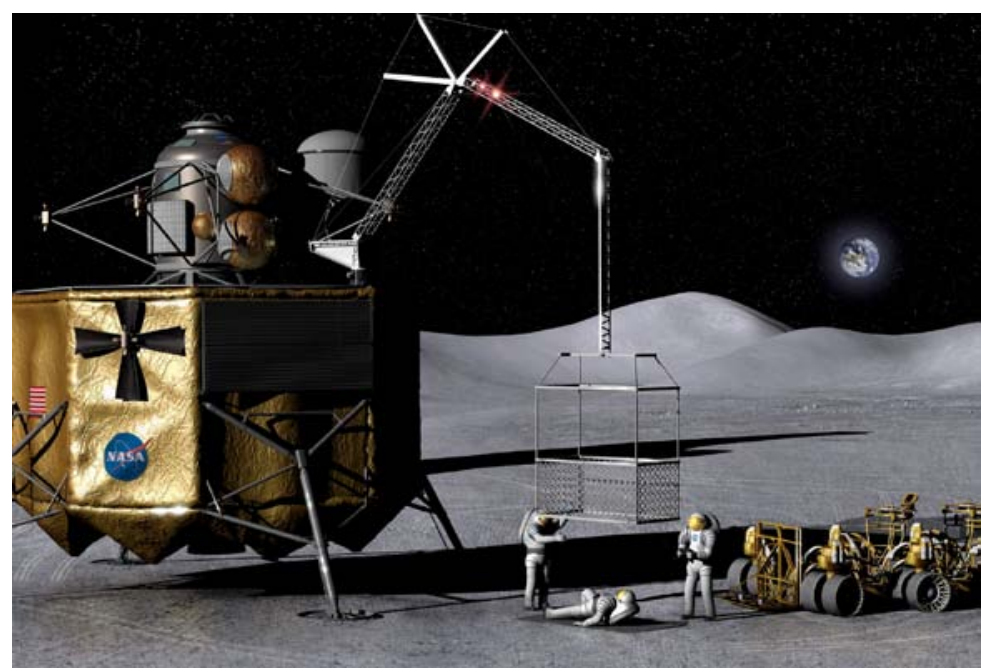

Figure 6. Single link LSMS on crewed lunar lander. incapacitated crew and a helper) enter the basket which is then lifted from the surface and placed in front of the airlock hatch. The total mass lifted in this scenario (basket plus $2 \mathrm{crew}$ ) is 600kg. In the second scenario, a small (Apollo class) rover (with mass of 210kg) is lifted from the payload deck and lowered to the surface. Operational reach studies, where the base of the LSMS was placed at several different locations on the lander deck, were conducted to determine the height of the LSMS king post and the length of the single link arm. Simplified models of the rover and the rescue basket were placed at initial storage locations on the lander deck and the LSMS link dimensions required to enable each operational scenario were determined. The storage location for the rover was centered in the clear area behind the ascent module. The LSMS was assumed to have a lifting link attached to the wrist for grappling the payloads and providing additional vertical reach for the arm. The height of the lander deck above the planetary surface was assumed to be 6.2 meters for this study. 
Two variations of the LSMS were structurally sized; one where the king post and arm link are assumed to be square tubular members, and one where they are assumed to be trusses. The LSMS sizing equations from Reference 2 were modified to account for the single link configuration and used to calculate link member lengths and the total LSMS mass. Three different combinations of initial LSMS base and basket storage locations were sized, as shown in Figure 7. Summary information for all of the sizing cases is given in Table 3. The placement location shown in Figure 7c resulted in the minimum mass single link LSMS. However, locating the base of the LSMS as shown in Figure 7a results in less than a $0.5 \mathrm{~kg}$ increase in mass, while positioning the base of the LSMS at a node point of the lander structure (where several struts are joined), which would serve as a very sturdy foundation point. In all cases, the mass of a single link LSMS, which can perform the intended operations, is shown to be very small.

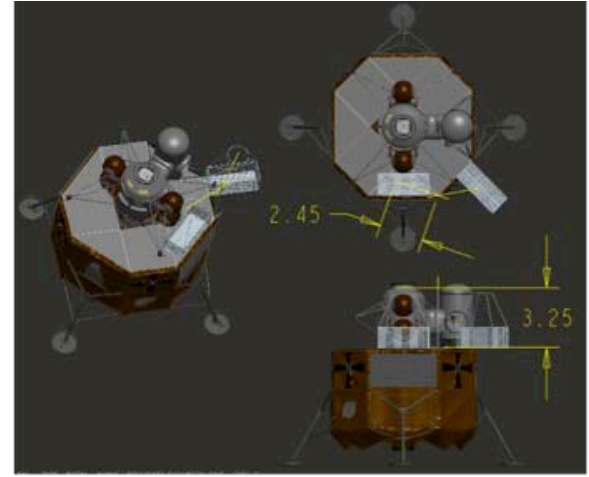

a. Elevator Placement Position 1.

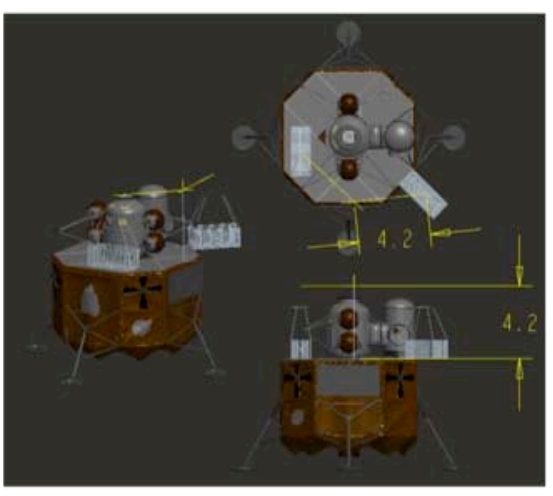

b. Elevator Placement Position 2.

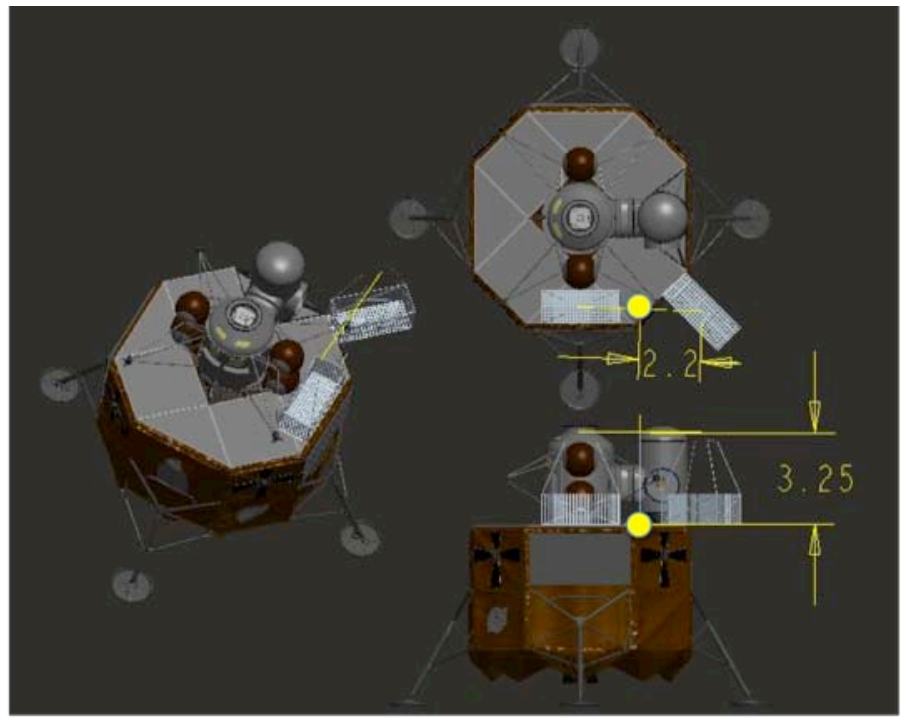

c. Elevator Placement Position 3 (chosen).

Figure 7. Single-link LSMS study configurations.

Table 3. Dimensions and masses for single-link LSMS configurations.

\begin{tabular}{|c|c|c|c|c|c|}
\hline $\begin{array}{c}\text { Placement } \\
\text { Configuration }\end{array}$ & Payload, kg & $\begin{array}{c}\text { King Post } \\
\text { Height, m }\end{array}$ & Arm Length, m & $\begin{array}{c}\text { Total Mass, } \\
\text { Tube Links, kg }\end{array}$ & $\begin{array}{c}\text { Total Mass, } \\
\text { Truss Links, kg }\end{array}$ \\
\hline $\begin{array}{c}\text { Basket-1 } \\
\text { (Fig. 7a) }\end{array}$ & 600 & 3.25 & 2.45 & 18.3 & 6.5 \\
\hline $\begin{array}{c}\text { Basket-2 } \\
\text { (Fig. 7b) }\end{array}$ & 600 & 4.2 & 4.2 & 32.5 & 11.0 \\
\hline $\begin{array}{c}\text { Basket-3 } \\
\text { (Fig. 7c) }\end{array}$ & 600 & 3.25 & 2.2 & 17.3 & 6.2 \\
\hline Rover & 210 & 2.1 & 2.1 & 7.1 & 1.8 \\
\hline
\end{tabular}




\section{Expanded Capabilities in the LSMS Test-bed}

The second effort, to continue expanding the versatility and autonomy of large planetary surface manipulators using the $1^{\text {st }}$ generation LSMS as a test-bed, is being accomplished by increasing manipulator capabilities and efficiencies. These are being advanced in two main areas: hardware modifications to increase manipulator dexterity along with new tools and end effectors; and, software and autonomous operations.

\section{A. Hardware Developments}

Several new hardware developments have expanded the operational capability of the LSMS test-bed, highlighting the device's versatility as introduced in Figure 8. Starting from the base and working outward, new hardware developments include: new shoulder joint and spreader hardware that increases the manipulator range of motion; a single degree-of-freedom tilt table, attached to the LSMS wrist to provide tools with the optimum orientation to perform a task; a robotic quick change at the wrist that provides mechanical and electrical connectivity and allows tools/end effectors to be automatically engaged and released; a forklift end effector to support pallet operations; change in geometry of the scoop end effector enabling it to be picked up with the quick change; and, a variable-length lifting link (VLLL) located at the wrist that can raise and lower payloads while preventing payload rotation. In addition, position sensors have

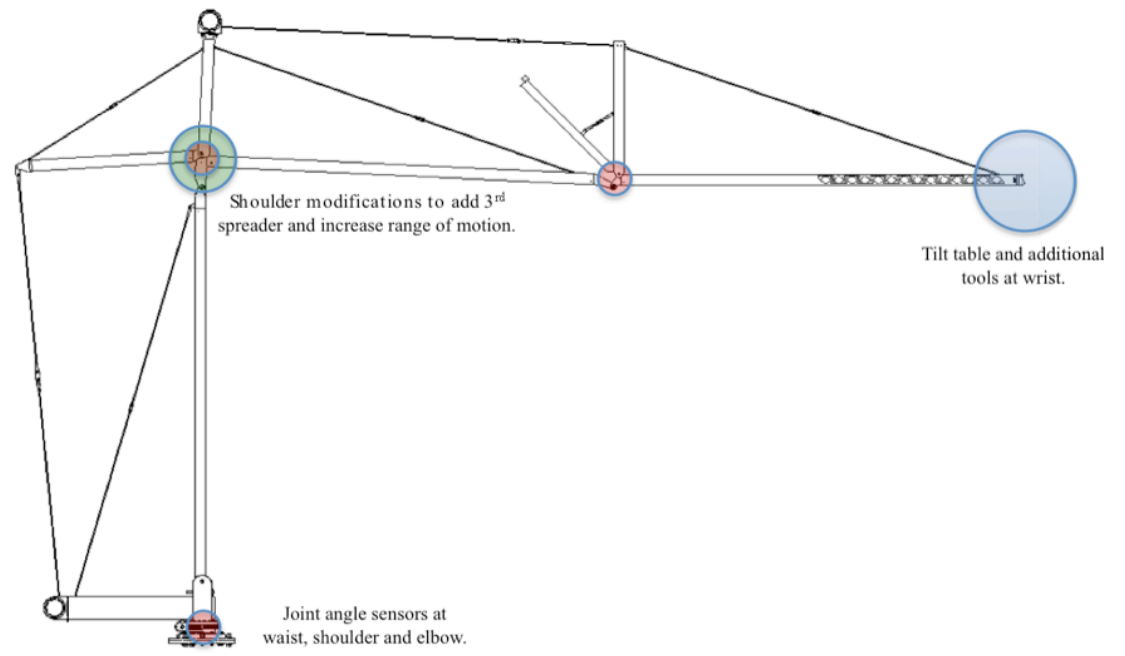

Figure 8. Locations of major new hardware developments on LSMS testbed. been installed at the waist, shoulder and elbow joints to allow end point control. The following sections provide details of these new capabilities and hardware.

\section{Shoulder Joint}

The shoulder was modified to achieve several objectives including: increasing the joint range of motion, improving the joint stiffness, enabling the kingpost to tilt forward and align the kingpost support diagonal with the pivot axis. The 1st generation joint design is shown in Figure 9a with the modified $2^{\text {nd }}$ generation joint design shown in Figure 9b. In Figure 9b, sensors are visible in yellow and black below the arm, just above and to the right of the figure center. The sensors, discussed in a previous paper $^{4}$, are not discussed here. 
For some operations, it is beneficial to lean the kingpost forward to extend the LSMS reach or increase the force that can be applied radially (from the base). For example, higher radial force is desirable when excavating with the shovel tool. As the king post leans forward, the angle between the kingpost and support diagonal changes. To accommodate this change in angle and have the line of action of the king post diagonal intersect the joint rotation axis, a long slot in the kingpost would be required. However, the slot in the kingpost can become so long that it compromises the torsional stiffness of the kingpost. To minimize the slot length, a bend in the diagonal was created using a short linkage; shown in Figure 9b as a gold rod through the cross section terminating in the light blue support component on the king post. The pivot axis is shown in red passing through the clevis, rod end and light blue support components. The location of the light blue support can be adjusted so that the diagonal is straight when the load in the diagonal is at its maximum.

The shoulder joint range of motion was increased by modifying the joint components and adding a third spreader, as shown in grey in Figure 9b. The operational joint range relative to horizontal increased from 45 degrees up/35 degrees down to 45 degrees up/75 degrees down without compromising packaging. The total physical joint range of the shoulder is much larger, having a range of 105 degrees up/90 degrees down, but requires disconnecting some tension rods and linkages that currently restrict motion. All three spreaders package against the arm, nesting together as they are packaged. Joint stiffness was improved by using offset fingers (see Figure 9b), as opposed to the more traditional clevis design used in the original configuration. This equalized the span on both sides of the joint, significantly improving the joint stiffness.

\section{Tilt Mechanism}

Attaching end effectors directly to the LSMS at the end of the forearm is not always desirable because the end effector orientation is then fixed relative to the forearm. A prime example of where this approach results in limitations is with the fork-

lift tines. The tilt mechanism was developed out of this necessity to have an additional rotational degree of freedom at the wrist. The tilt mechanism consists of a pivoting mounting plate, shown in blue in Figure 10, with a set of fixed arms

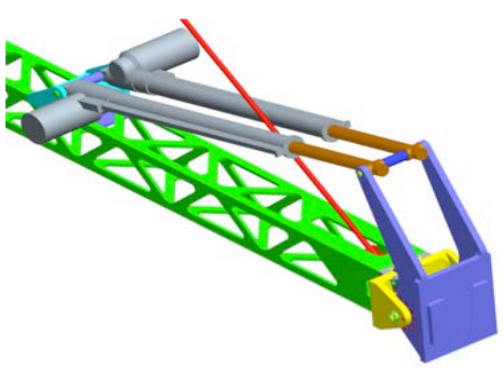

extending upward that attach to two parallel linear actuators. The tilt mechanism provides \pm 45

degrees of motion relative to the forearm. The tilt mechanism currently includes a limit switch to control extension and retraction stops that allows operational development. The limit switch is mounted in-between the fixed and rotating pivot plates and is actuated by a replaceable cam (Figure 11). The cam is easily changed so that ones with different stop settings can be installed to adjust the tilt mechanism range of motion. The tilt mechanism has been installed and its full range of motion has been operationally verified. A tilt sensor will be installed in the near future.

\section{Honeybee Tool Quick Change}

Figure 12 depicts the original LSMS wrist connection, which uses a mechanical interface that combines a tapered $\mathrm{V}$ groove with a dovetail so that end effectors can be easily attached and removed without the need for tools. A push button release pin is incorporated as a safety lock. The device was sized for the full payload capacity of the LSMS operating in 1g; $150 \mathrm{~kg}$ payload that is offset $0.75 \mathrm{~m}$ out from the end of the forearm.
Figure 11. Replaceable cam for tilt mechanism.

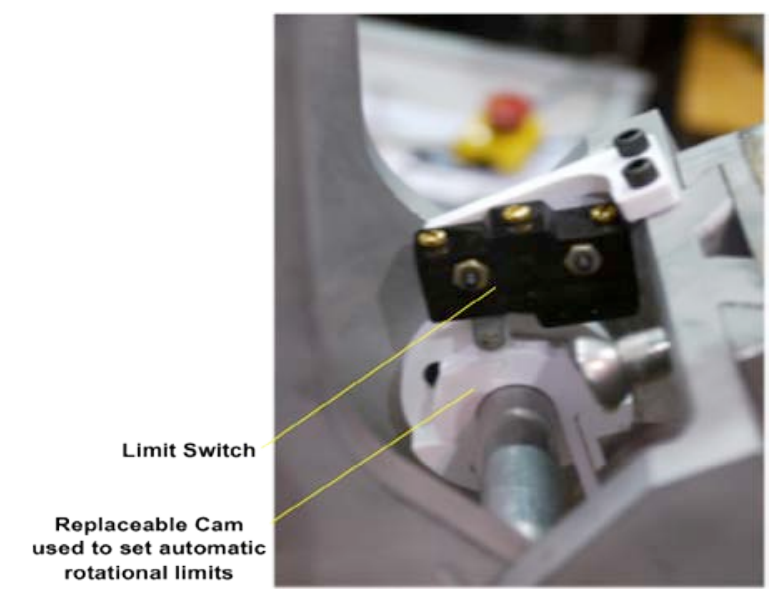


In order to further automate LSMS operations, a capability was needed to replace the manual process for changing tools and end effectors. A robotic tool "quick change" device (a term originating from the world of manufacturing automation) is a special end effector, mounted permanently to the robot wrist and allows a robotic arm to change grippers, tools or instruments automatically (unassisted by humans). A complete robotic quick change typically provides a means for making mechanical, electrical and sometimes fluid connections. The ability for a planetary surface robot, such as the LSMS, to perform unassisted tool change operations greatly increases the manipulator's utility since it allows the robot to accomplish many different tasks

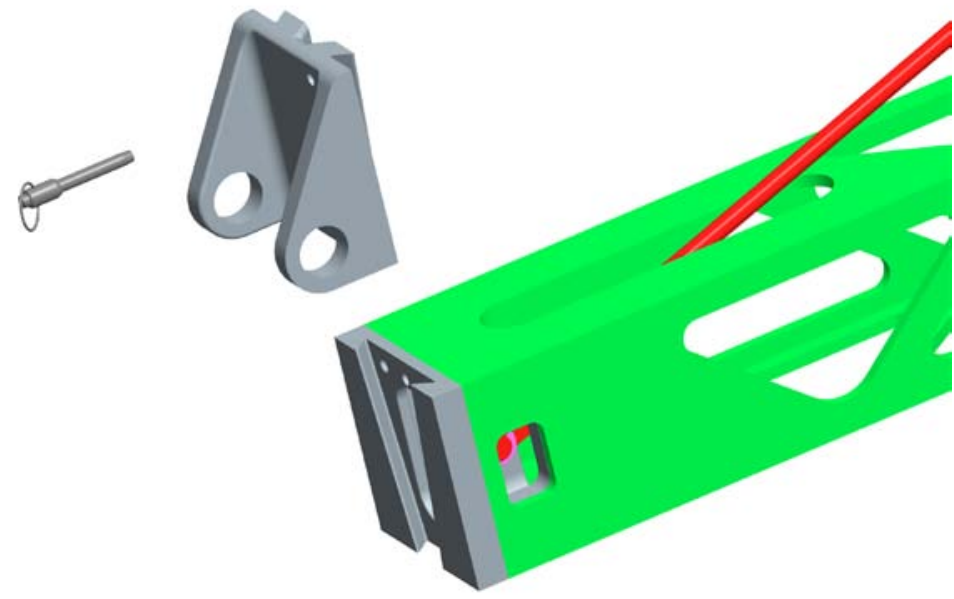

Figure 12. Original end effector connector at the wrist.

without requiring intervention by an astronaut. Due to the harsh nature of lunar dust and regolith (very abrasive, adhesive and cohesive) a robotic quick change for use on the lunar surface must include dust tolerant features in its design as well as in the interfaces used in making mechanical and electrical connections.

Honeybee Robotics developed dust tolerant electrical connectors under a Phase 1 Small Business Innovation Research (SBIR) contract with NASA Glenn Research Center. Based on their SBIR results and experience with tools and hardware operations on the Mars surface, the LSMS team has funded two successive Phase 3 SBIRs with Honeybee to develop a mechanical robotic tool quick change for planetary surface payload handling operations. The challenge was to develop a device that could repeatedly and reliably operate in an extreme temperature and dust environment while making a mechanical (resisting forces and torques) and electrical connection to a variety of robotic tools and end effectors. The first SBIR focused on the overall device configuration, operation concept, design and fabrication. The resulting quick change was capable of making only the mechanical connection. The second SBIR was awarded to incorporate the Honeybee electrical quick change connector and a complete system is currently being fabricated.

The robotic quick change is currently operating on the $1^{\text {st }}$ generation LSMS test-bed, but was designed to be compatible with the increased payload capabilities of the $2^{\text {nd }}$ generation LSMS. The quick change design requirements included; supporting a $500 \mathrm{~kg}$ payload, a $250 \mathrm{~kg}-\mathrm{m}$ moment and accommodating vertical and lateral misalignments of \pm $2.54 \mathrm{~cm}$ (shown in Figures 13a and 13b respectively) and 10 degrees (about a vertical axis), as shown in Figure 13c. The final design of the 1st system, shown in Figure 14, consists of an active drive unit that is attached to the LSMS

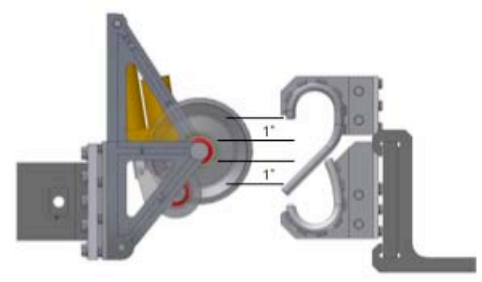

a. Vertical misalignment.

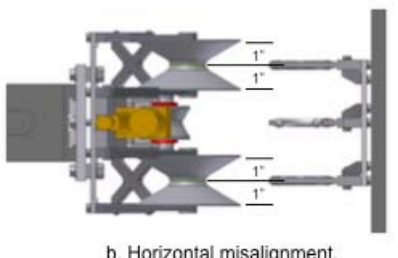

b. Horizontal misalignment.

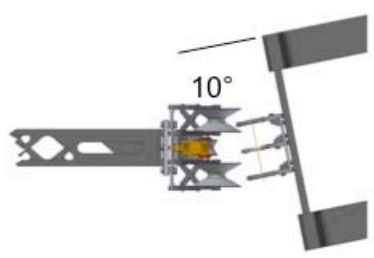

c. Rotational misalignment.

Figure 13. Allowable misalignment ranges for quick change.

wrist and a passive plate/hook system that is attached to every tool and end effector that will be grappled. The design also incorporated the following features (as noted in Figure 14) for dust mitigation: lip seals for dust resistant rotational joints; three points of rolling contact and large clearances to prevent dust from causing mechanical friction and wear; rotating alignment wheels to reduce failure due to dust-induced galling or seizing, and to allow easy separation; capability (future) to seal the linear actuator with a bellows for dust protection. 


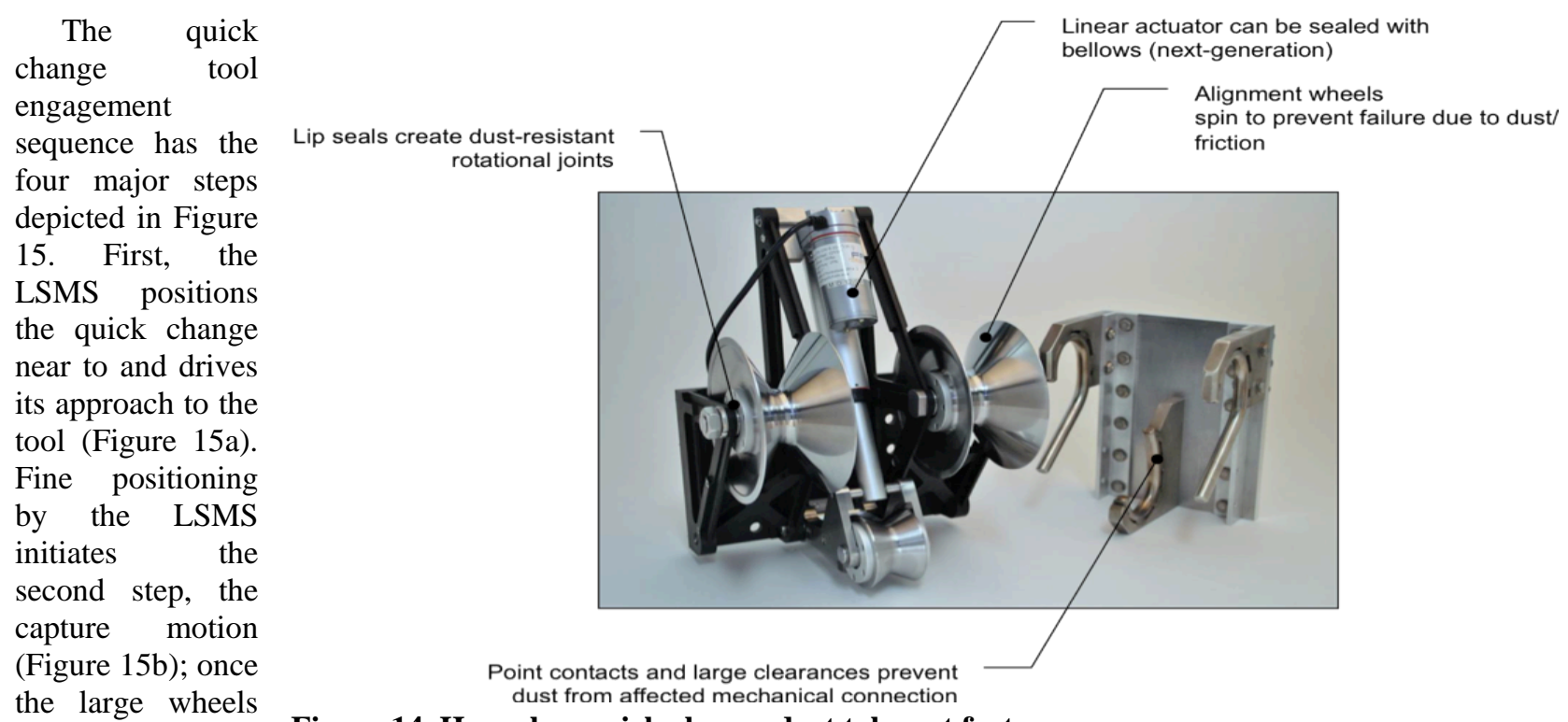

of the active side

come into contact

with the hooks on the passive side, the 30-degree slopes of the wheels guide the rolled round hooks into position. In step three, soft capture occurs and the tool/end effector is lifted slightly with either pure vertical or large-arc motion

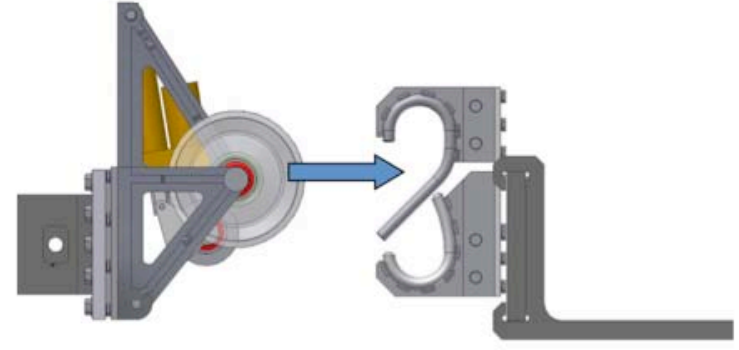

a. Approach tool.

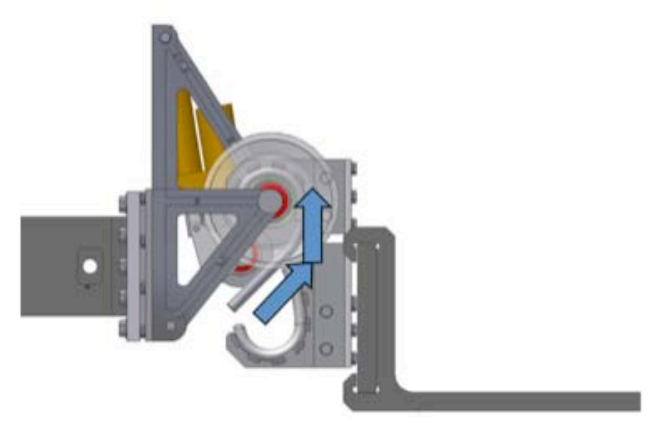

c. Soft capture and lift tool.

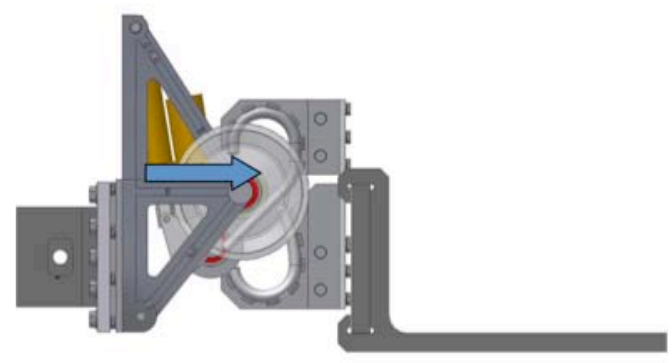

b. Begin capture motion.

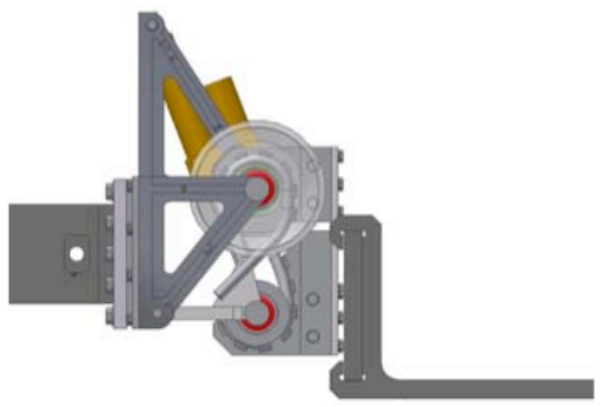

d. Lock tool in place with actuator.

Figure 15. Honeybee quick change operational sequence.

(Figure 15c). In the fourth and final step, the actuator drives the small wheel into contact with its associated hook (on the passive half), locking the tool in place (Figure 15d). The procedure is reversed to release the tool.

The active side of the quick change encompasses a volume of 14.0 inches height by 9.4 inches width by 9.6 inches depth (which includes the LSMS adapter plate). The actuator can apply $150 \mathrm{lbs}$ of force and is powered by a 12-volts DC motor through a control box. The mass of the quick change active side is $13.9 \mathrm{~kg}$ and the mass of a passive side (which must be attached to each tool) is $2.7 \mathrm{~kg}$. The quick change can be used to directly grapple 
payloads if the payload has a passive hook side attached. This was demonstrated in the first use of the quick change system, where an adapter plate with a passive hook side was attached to the side of a ISRU payload, as shown in Figure 16a. A check-out demonstration was successfully completed where the ISRU payload was automatically grappled, lifted, placed on a platform and then released (see Figure 16b). Alternatively, any one of many tools can be grappled and each tool will provide a different mode of payload handling. As an example, a passive hook side

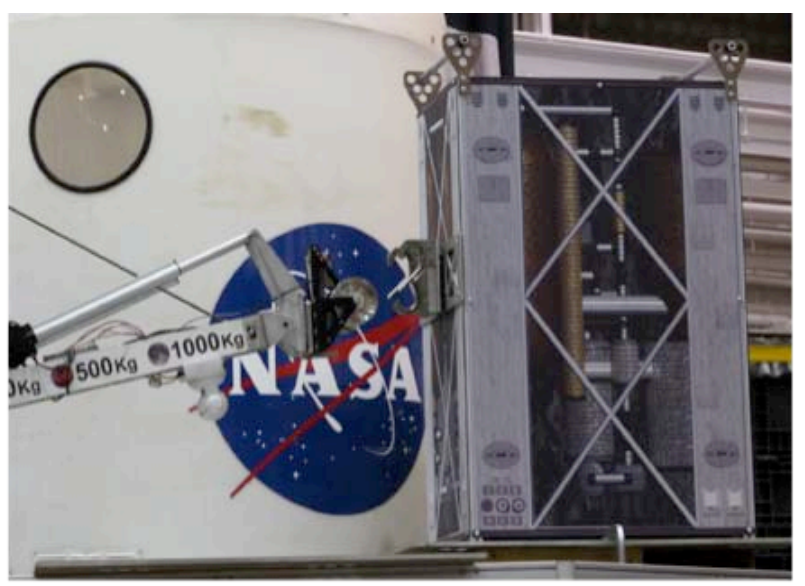

a. Direct grappling of payload.

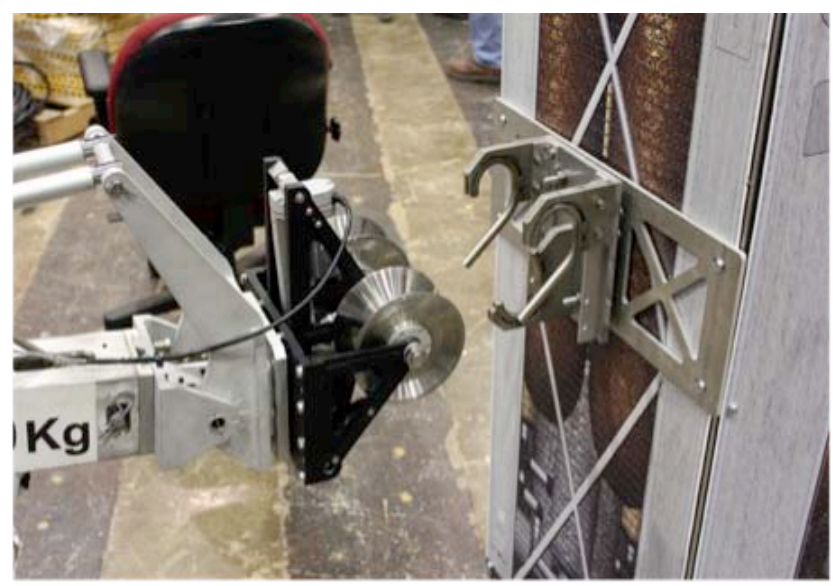

b. Automatically releasing payload.

Figure 16. Operations using Honeybee quick change.

was also attached to the fork lift tine tool, and the LSMS was able to automatically grapple the tool and unload a pallet from the lander mockup (discussed in subsequent section, see Figure 21c).

4. Overhead Lift and Variable-Length Lifting Link

In contrast to a typical crane, the LSMS is a hybrid crane manipulator that supports all types of possible methods for manipulating payloads including: overhead hook operations typically thought of when cranes are discussed; side grapples (like a robotic manipulator), and; pallet operations, where the payload is lifted as is done with a forklift. Tools to accomplish these operations will be discussed in this and the following three sections.

The LSMS is able to pick up objects at both the wrist and elbow, as shown in Figure 17. The capacity at the elbow is approximately twice the wrist payload due to the reduced moment at the shoulder. Initial operations with fixed-length lifting links, as shown in the figure, have been very successful. Unlike a cable, the lifting links do not twist and thus provide significant control authority over the payload allowing precise autonomous positioning. Lifting links are designed to compactly package at either the wrist or elbow by folding against the forearm or arm respectively.

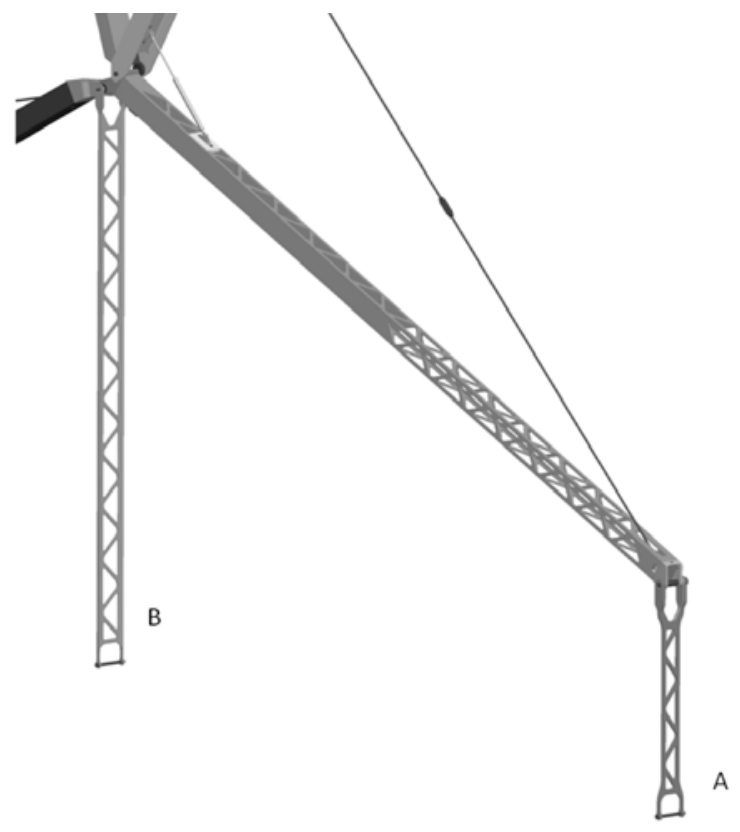

To expand the lift range at the wrist while still Figure 17. Lifting locations at LSMS elbow and wrist. maintaining the twist resistant characteristic of the lifting link, a Variable-Length Lifting Link (VLLL) has been designed as shown in Figure 18. In order to minimize cost and development time, a cable-driven hoist (WARN Pullzall ${ }^{\circledR}$ ) was chosen to actuate the VLLL (see Figure 18). The hoist working load rating of $1000 \mathrm{lb}$ is considerably larger than the $330 \mathrm{lb}$ payload rating at the LSMS wrist, providing a large operational margin of safety. The upper end of the VLLL consists of guided rollers that allow the link to travel up and down as the hoist reels cable in and out. On the bottom end of the VLLL, attached to the bottom of the hoist, is a short fixed-length of link 
with a cutout and shaft that can engage the standard hook (hook shown in Figure 19) attached to the top of most payloads used in operational tests.

The total length of the link is $3.5 \mathrm{~m}$. Two simple brackets were designed and fabricated and are used to attach the hoist to the VLLL draw bar. Mechanical hard stops are located at both travel extremes of the VLLL, to prevent the draw bar from dropping out of the upper guides or colliding with the hoist on the lower end. Limit switches are being installed to halt actuation before the stops are reached. The trigger of the hoist is being modified to enable proportional control using an Arduino® microcontroller. This will enable automated operations or remote tele-operated control of
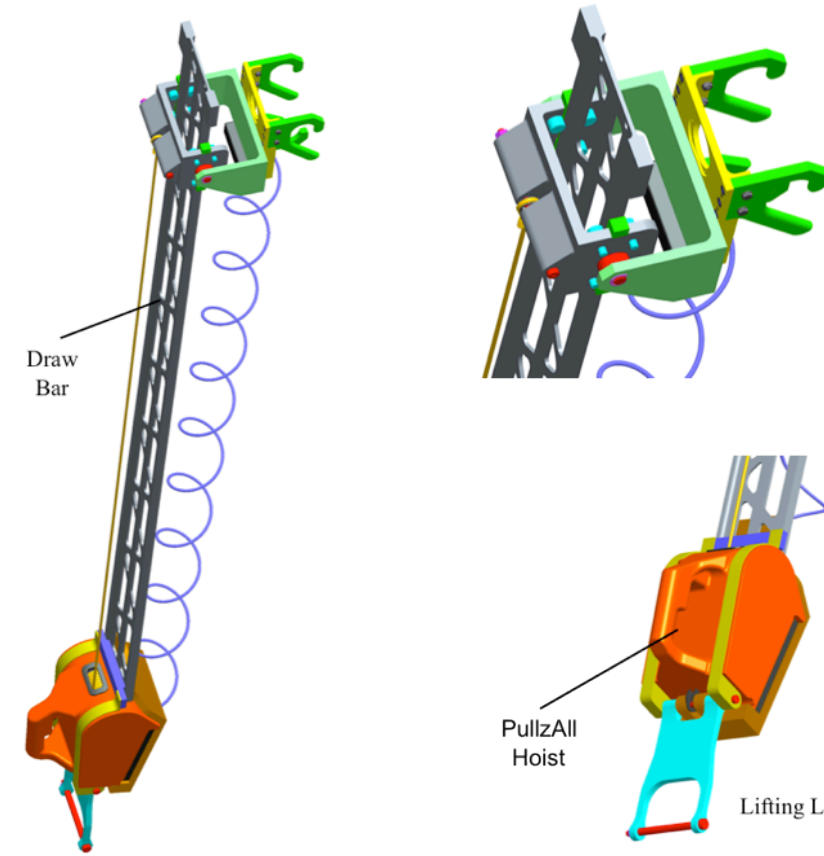

Roller Assembly and Quick Change Passive Side the VLLL.

5. Side Grapple

A gripper is a common tool for robotic manipulators. Taking advantage of an existing device shown in Figure 20, custom fingers were designed and fabricated to be compatible with existing International Space Station micro-conical fixtures. The gripper was further modified to include a central guide pin to locate the fixture and provide a visual aid during tele-operated capture operations. The fingers are actuated by jaws that travel on linear bearings, which in turn, are actuated by a double helical spiral.

\section{Pallet Operations - Fork Lift Tines}

One of the more common material handling devices is the fork-lift. The large range of motion of the LSMS enables it to execute motion similar to a forklift using the tines shown in Figure 21. Figure 21a shows the tines (in yellow) integrated with

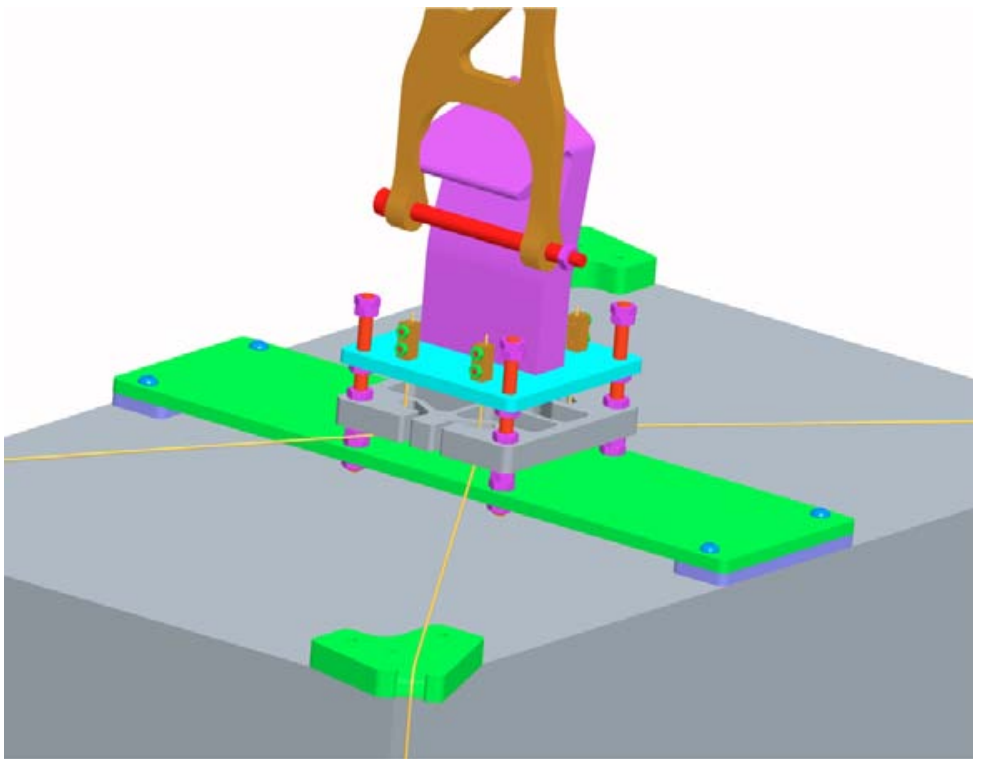

Figure 19. Lifting link engaging standard payload.

the tilt table and quick change components. Figure $21 \mathrm{~b}$ is an exploded view showing all of the subcomponents. The tines were designed to support a $150 \mathrm{~kg}$ payload at an offset distance of $0.5 \mathrm{~m}$ from the backing plate. The tine spacing is manually adjusted by removing a locking pin, sliding the tines to a new position, and reinserting the locking pin. During operations (Figure 21c), the mass of the lifted payload is nominally centered between the tines to prevent undesirable torques on the forearm. Limits on off-axis loads have not been quantified at this time.

7. Digging Operations - Shovel

In order to meet anticipated needs to excavate, place and trench regolith, a shovel tool was developed. Three operational tasks were identified to help determine design parameters. In the first task, berms are formed between habitation and landing zones; in the second task, a habitat is covered with regolith for galactic cosmic radiation protection; and in the third task, regolith is excavated and used to shield nuclear reactors. The reactor task was further divided into two scenarios; one where the reactor was placed in an excavated trench and covered with 
regolith (note the LSMS is an effective device for positioning the reactor and connecting required cabling), and a second, where the reactor was covered with regolith (before the reactor is activated) while remaining on the lander. To execute these tasks, a shovel (see Figure 22) was designed that could lift a mass of $150 \mathrm{~kg}$ of regolith (when fully loaded), equal to the payload capacity at the LSMS wrist. Tests using dry sand with the shovel directly attached to the wrist resulted in the shovel scooping, on average, approximately $70 \mathrm{~kg}$ of material. This was lower than expected due to the inability to tilt the shovel upward as the shovel was raised, which allowed a significant amount of material to escape. There are plans to reevaluate the shovel's capability with it attached to the tilt unit, as shown in Figures 22a and 22b.

A single solenoid is used to release a spring actuated latch as shown in Figure 23. Figure 23a shows the latched bucket prior to

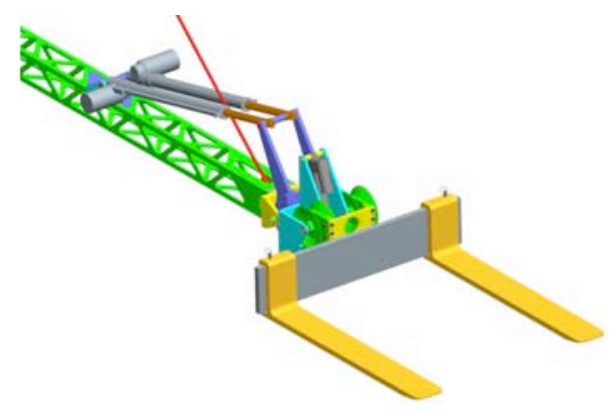

a. Integrated system.

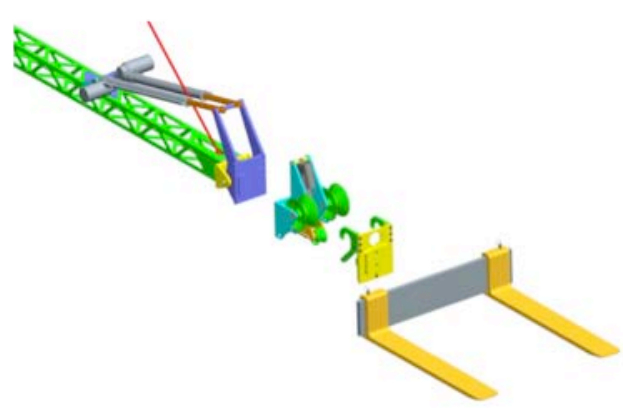

b. Exploded view showing components.

Figure 21. Fork lift tines.

release, while in Figure 23b the solenoid has been activated to release the bucket, causing it to pivot and dump its load. To reset the shovel, the LSMS is used to slide the shovel backwards against any suitable surface until the top mounted spring-loaded latch is re-engaged.

American Institute of Aeronautics and Astronautics 


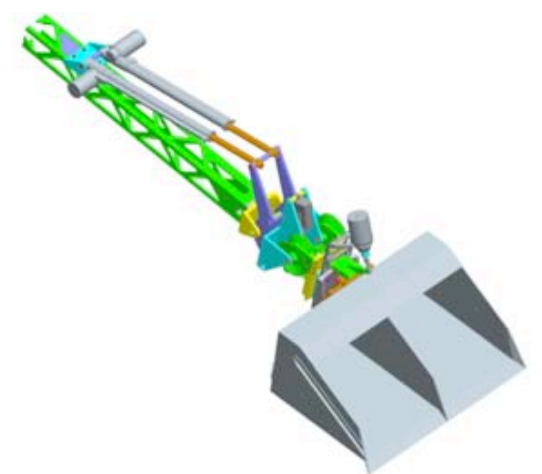

a. Integrated system.
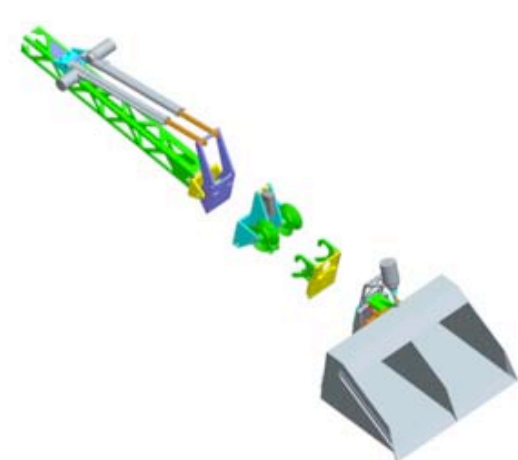

b. Exploded view showing components.

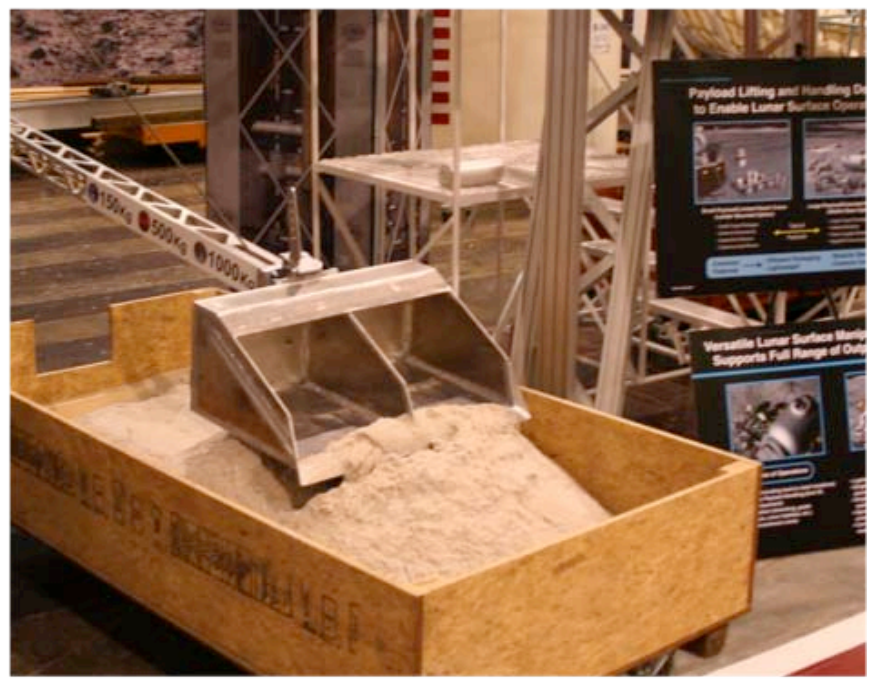

c. Hardware in operation.

\section{Figure 22. Shovel end effector.}

\section{B. Operations and Software Developments}

1. Vision and Tracking System

The objective of the software development effort is to expand the operational capabilities of the LSMS test-bed to include autonomous operations based on stored paths. This is the first step toward integration with a path planner to enable execution of tasks based on geometry models. The sensor system and kinematics developed in Reference 4 have been implemented and verified. Proportional Integral Derivative (PID) control loops have been implemented that use the sensor signals to rotate each LSMS joint to its desired joint angle, but the link with preplanned Cartesian paths is still being developed.

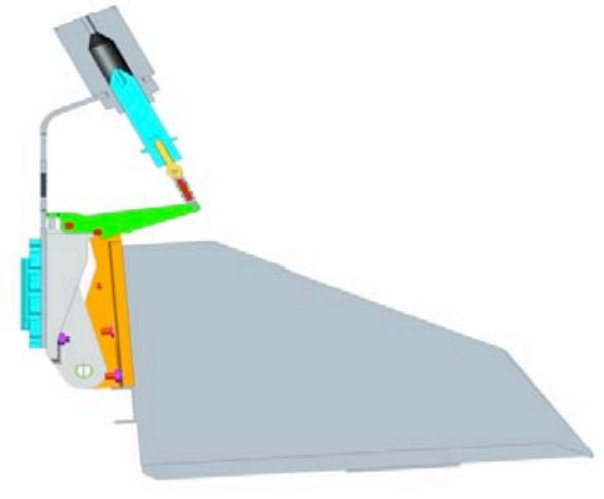

a. Latched

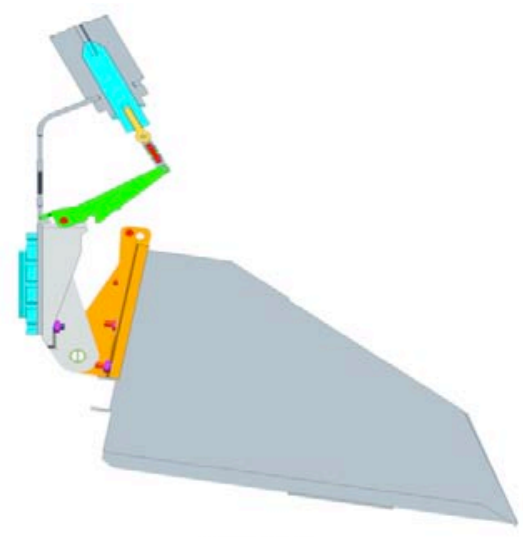

b. Released.

Figure 23. Shovel bucket automatic release system.

One of the first applications of autonomous capabilities will be to integrate a machine vision system that enables automated identification and grappling of payloads by using passive targets that are affixed to the payloads. The machine vision system provides the location of the payload relative to the camera, which is fixed to a known location on the LSMS. Using this location information, the LSMS is directed to a position where either the payload can be grappled from the side or lifted with a lifting link. 


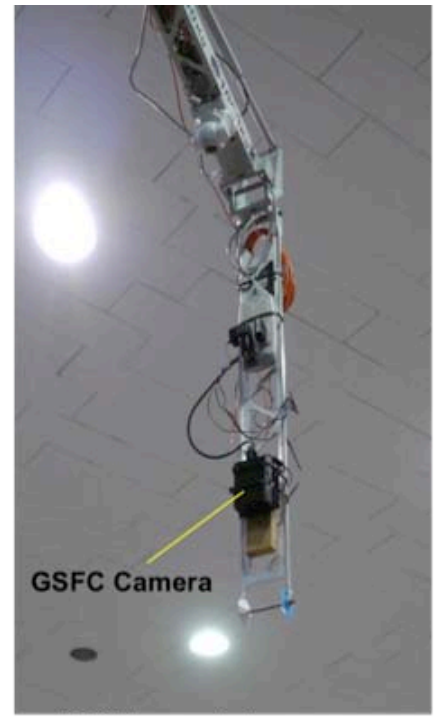

a. GSFC Camera System Mounted to LSMS Wrist

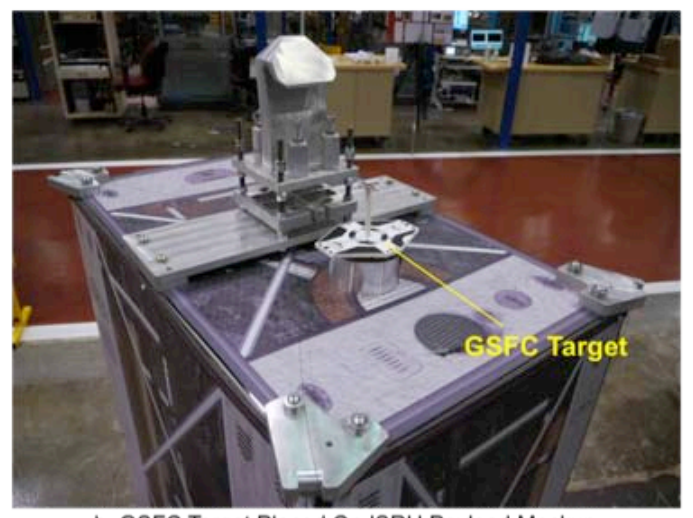

b. GSFC Target Placed On ISRU Payload Mockup

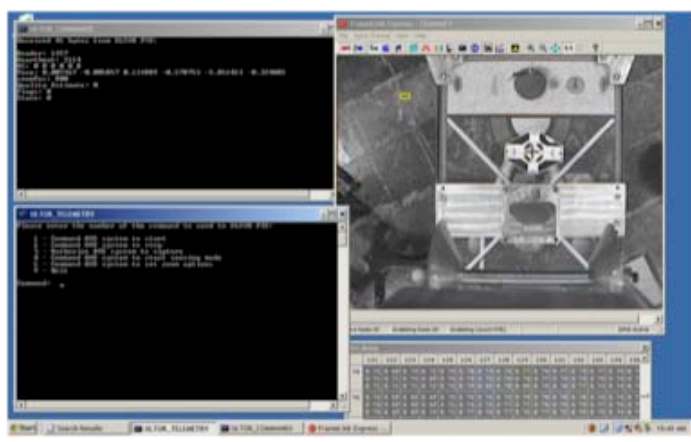

d. Screen Shot From Lap Top Control Computer

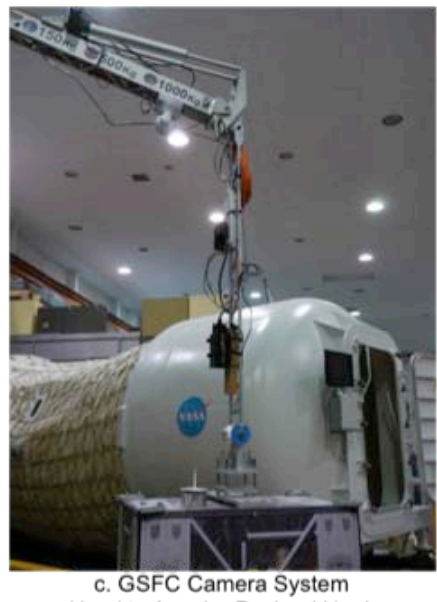

Used to Acquire Payload Hook

\section{Figure 24. Setup to test Ultor vision and tracking system.}

The machine vision system is currently on loan from colleagues at the Goddard Space Flight Center (GSFC). The system was supplied to GSFC by Advanced Optical Systems, Inc. and is called the Ultor Vision System ${ }^{5}$. It consists of a Passive Pose Position Engine $\left(\mathrm{P}^{3} \mathrm{E}\right)$, camera and host computer. The Ultor system is queried by the host computer when the pose information associated with a predefined passive target is desired. The basic Ultor system commands are start, stop, track and quit. The start command sets the Ultor to default values found in its internal parameter file and stop tells the Ultor to cease tracking. The track command uses the camera to acquire a visual image of the environment from which it extracts the target, then it acquires and continuously tracks the target as it moves in the field of view. The quit command is used to terminate a session; stopping tracking and closing the TCP connection between the Ultor and host computer.

The GSFC camera system was installed on a lifting link with the aperture pointing down to look for a target below (Figure 24a). A standard target was placed on top of the ISRU payload mockup, as shown in Figure 24b. While the Ultor system is capable of acquiring a target at significant range, operations with the LSMS test-bed require pose (position and orientation) information of the target over a range of 2 to 6 meters and operation in the 2 to 6 meter range has been verified using the setup shown in Figure 24c. Several experiments were completed that successfully demonstrated; acquire the target at different separation distances, tracking the target as the LSMS wrist moved vertically, and using video output to the computer display (Figure 21d) to help engage the hook on the payload.

\section{Remote Operations}

A major goal of the LSMS project for fiscal years 2009 and 2010 was to integrate the LSMS into the HRS remote control infrastructure, known as the Robot Application Programming Interface Delegate (RAPID) system, and develop the capability to operate the LSMS from a remote site. The goal of the multi-center RAPID project is to create a common operations system capable of monitoring and commanding all elements of the HRS architecture ${ }^{6}$. This system incorporates planning, scheduling and supervising of operations from a remote location, can integrate imagery and data from multiple assets, and will act as the focal point for communication and interaction between operators, robots and in-situ personnel. The RAPID workbench includes several components or 'clients' to perform its' functions (Figure 25a). The Jet Propulsion Lab is programming the primary Mapping and Telemetry Canvas, which will track and display all Human Robotic Systems (HRS) project elements and their respective locations, as 
well as provide a Graphical User Interface (GUI) for telemetry input and output. Johnson Space Center's Predictive Interactive Graphical Interface (PIGI) provides robustness of communication over intermediate time delays by calculating and predicting where an asset will be and displaying that path graphically so that preemptive control inputs can be made. Lastly, Ames Research Center has developed a software package called 'Viz' to graphically display the configuration of each HRS asset in real-time using fully articulate 3D models.

In order to integrate the LSMS into the RAPID architecture, the command and monitoring interface currently used for control of the LSMS, and written in LABVIEW ${ }^{\circledR}$ must interface with the RAPID API. The RAPID team programmed a simple bridge that takes a set of standardized parameters from the LSMS LABVIEW ${ }^{\circledR}$ application, translates them into RAPID protocol, and then receives updated parameters back over the bridge (Figure 25a). The 'Message Set' of data from the LSMS can include the following: Status, power and current task; Position, location and orientation; Joint Data, angle and motor velocity; and Picture Data, images and video. The return 'Command Set' from RAPID includes: Get Status, requests some or all of the above parameters; Robot Commands, change joint angles and velocities; and Camera Commands, such as orient cameras, take picture and/or video. A remote operator is provided with a workbench environment called the Multi-Center Cockpit (MCC), which can send and receive the data sets to and from the assets and display all available telemetry simultaneously.

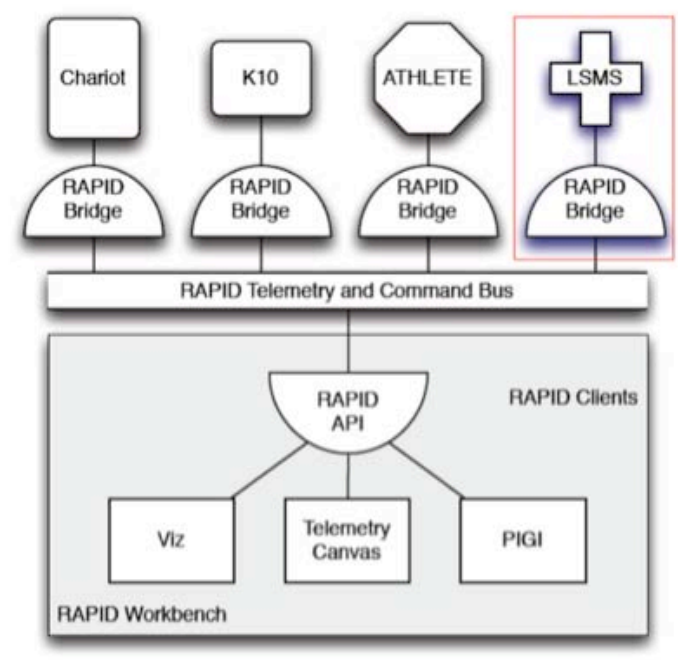

a. RAPID Command hierarchy and components.

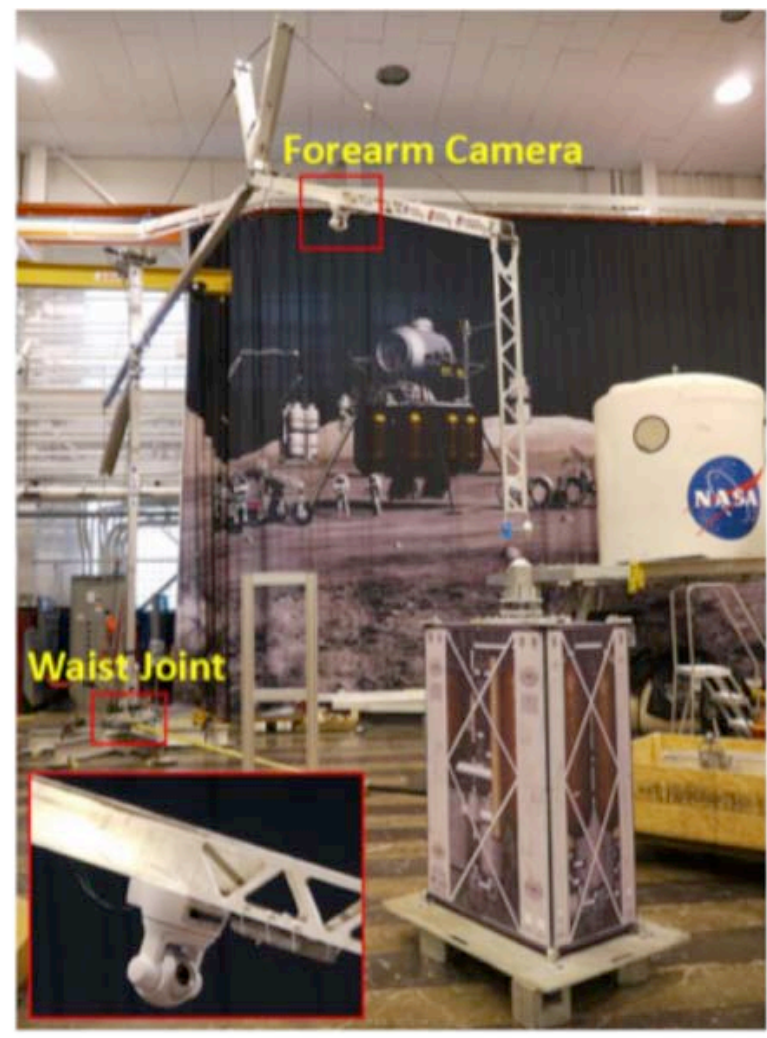

b. LSMS components controlled over RAPID bridge.

Figure 25. RAPID communication system flow.

The LSMS team has successfully run a series of trials with the RAPID software bridge that implemented and demonstrated the use of the robot and camera commands. The first tests sent and received three joint angles across the bridge demonstrating its function. Portals were opened up on both the JPL and LaRC network firewalls to allow the communication to occur. The joint angles were input at JPL on the MCC and were displayed on the control GUI of the LSMS, where updated angles were then sent back to JPL. The joint angles for this first test were produced by manually actuating a set of bench mounted joint angle sensors. The second set of tests had an operator at JPL command the waist joint of the LSMS to several different angles using the RAPID control commands while monitoring the position via a live video feed from the forearm camera of the LSMS (Figure 25b). The JPL operator also demonstrated panning, tilting and zooming the camera as desired during the test. For the second set of tests, only one of the joint angle sensors was mounted; at the waist joint. Angle sensors have since been installed at the shoulder and elbow of the LSMS so that all three angles can now be sent and received. With the current protocol, a 
remote operator also has access to up to four camera views, although presently there are only two mounted on the LSMS and one mounted in the lab adjacent to the manipulator.

With the addition of joint angle sensors and the integration of the LSMS into the RAPID architecture, remote semi-automated and automated control becomes a reality. The inverse kinematics of the 3 degree-of-freedom system have been determined and will be used to calculate the joint angles to place the LSMS wrist at a given location in 3D space. Currently the motor velocities are controlled independently in manual mode, but with the joint angles known, cartesian positioning control is now being added as an option. This allows the operator to use a single forward command, for example, where previously they would have had to drive the shoulder and elbow motors separately. Future work will incorporate path planning and hazard avoidance using the onboard cameras to fully automate sequenced operations such as searching for, engaging and offloading payloads.

\section{Concluding Remarks}

The 1st generation of a versatile new high performance device for performing payload handling and assembly operations on planetary surfaces, the Lightweight Surface Manipulation System (LSMS), has been designed and built. Over the course of its development, conventional (crane type) LSMS payload handling configurations and operations were successfully demonstrated during field tests at Moses Lake, Washington. Subsequently, the range of motion, types of operations and the versatility of the LSMS have been greatly expanded and this updated set of $1^{\text {st }}$ generation LSMS hardware is now serving as a laboratory test-bed to continue to develop end effectors, operational techniques and remotely controlled and automated operations.

The most recent LSMS and test-bed development activities have focused on two major efforts. The first effort was to complete a preliminary design of the $2^{\text {nd }}$ generation LSMS that has the capability for limited mobility and can reposition itself between lander decks, mobility chassis, and fixed base locations. A major potion of this effort involved conducting a study to establish the feasibility of and define the specifications for a lightweight cable-drive waist joint. The second effort was to continue expanding the versatility and autonomy of large planetary surface manipulators using the $1^{\text {st }}$ generation LSMS as a test-bed.

The complete set of features, changes and improvements made in evolving the LSMS from its first to its $2^{\text {nd }}$ generation implementation is summarized. This results in an improved LSMS that can lift three times the payload of the 1st manipulator, yet have the same total device mass. Key to the success is developing a lightweight cable-drive waist joint and two independent studies were conducted that established the viability of a lightweight cable drive waist joint design. The $2^{\text {nd }}$ generation design versatility was demonstrated by using it as the basis to design a single link version of the LSMS. This very lightweight version was successfully demonstrated to solve the critical need for lifting incapacitated crew from the surface of a planetary surface, to the top of the lander deck, allowing the injured crew to be positioned inside an ascent stage for either emergency medical care or evacuation.

The second major effort, research into increasing the versatility and autonomy of large planetary surface manipulators using the $1^{\text {st }}$ generation LSMS as a test-bed, is being accomplished by increasing manipulator capabilities and efficiencies. The test-bed shoulder joint was redesigned and a spreader added that greatly increased the LSMS range of motion. A tilt mechanism was added to the wrist that provides increased dexterity for tools and end effectors. A dust tolerant (for robust planetary surface operations) robotic quick change mechanism, that allows tools and end effectors to be changed automatically, has been developed and attached to the tilt table. The robotic quick change also has the capability to directly grasp payloads that have a passive hook side mounted to them. A comprehensive set of end effectors that include; a variable-length lifting link, a gripper, fork lift tines and a shovel, have all been designed and operationally tested.

The software development effort is expanding the operational capabilities of the LSMS testbed to include autonomous operations based on stored paths. This is the first step toward integration with a path planner, which will enable tasks to be executed based on geometry models. The joint sensor system and joint kinematics have been implemented and verified and Proportional Integral Derivative (PID) control loops have been implemented that use the sensor signals to servo each LSMS joint to its desired joint angle. A vision system was integrated and used to demonstrate target acquisition and tracking and final hardware integration with the LSMS and software integration with the closed form kinematics is being developed. A series of trials with the RAPID software bridge, that implemented and demonstrated the use of the robot and camera commands, was successfully run. The first tests sent and received three joint angles across the bridge demonstrating the function of the communication bridge.

\section{References}

1. Doggett, William R.; King, Bruce D.; Jones, Thomas C.; Dorsey, John T.; and Mikulas, Martin M.: Design and Field Test of a Mass Efficient Crane For Lunar Payload Handling and Inspection - The Lunar Surface Manipulation System. Presented at 
the AIAA Space 2008 Conference and Exposition, 9 - 11 September 2008, San Diego, California. Available as AIAA-20087635.

2. Dorsey, John T.; Mikulas, Martin M.; and Doggett, William R.: Preliminary Structural Design Considerations and Mass Efficiencies for Lunar Surface Manipulator Concepts. Presented at the AIAA Space 2008 Conference and Exposition, 9 - 11 September 2008, San Diego, California. Available as AIAA-2008-7916.

3. Dorsey, John T.; Jones, Thomas C.; Doggett, William R.; Roithmayer, Carlos M.; King, Bruce D.; and Mikulas, Martin M.: Developments to Increase the Performance, Operational Versatility and Automation of a Lunar Surface Manipulations System. Presented at the AIAA Space 2009 Conference and Exposition, 14 - 17 September 2009, Pasadena CA. Available as AIAA-2009-6795.

4. Doggett, William R.; Roithmayer, Carlos M.; Dorsey, John T.; Jones, Thomas C.; Shen, Haijun; Seywald, Hans; King, Bruce D.; and Mikulas, Martin M.: Automation of a Versatile Crane (the LSMS) for Lunar Outpost Construction, Maintenance and Inspection. Presented at the AIAA Space 2009 Conference and Exposition, 14 - 17 September 2009, Pasadena CA. Available as AIAA-2009-6546.

5. Joel Hannah, (2011, April 6). “Proposed Ultor Data Link,” Advanced Optical Systems, Huntsville, AL.

6. Torres, R. J., Allan, M. B., Hirsh, R. L., "Rapid Workbench: Results of Collaboration Between Three NASA Centers in Commanding and Monitoring Lunar Assets,” 2009 IEEE Aerospace Conference [submitted for publication], IEEE, New York, NY, Mar. 2009. 